\title{
Self-Assembly of a Filament by Curvature-Inducing Proteins
}

\author{
James Kwiecinski, S. Jonathan Chapman, Alain Goriely* \\ Oxford Mathematical Institute, Andrew Wiles Building, Woodstock Road, Oxford, OX2 6GG, United Kingdom
}

\begin{abstract}
We explore a simplified macroscopic model of membrane shaping by means of curvature-sensing BAR proteins. Equations describing the interplay between the shape of a freely floating filament in a fluid and the adhesion kinetics of proteins are derived from mechanical principles. The constant curvature solutions that arise from this system are studied using weakly nonlinear analysis. We show that the stability of the filament's shape is completely characterized by the parameters associated with protein recruitment and establish that in the bistable regime, proteins aggregate on the filament forming regions of high and low curvature. This pattern formation is then followed by phase-coarsening that resolves on a time-scale dependent on protein diffusion and drift across the filament, which contend to smooth and maintain the pattern respectively. The model is generalized for multiple species of BAR proteins and we show that the stability of the assembled shape is determined by a competition between proteins attaching on opposing sides.
\end{abstract}

Keywords: Self-Assembly, Membrane Remodeling, BAR Proteins

\section{Introduction}

Self-assembly is a ubiquitous phenomenon that exists over a large range of length scales in systems both physical and biological in nature. Examples range from the astronomically large, such as the formation of galaxies and planetary systems [10], to the nano-scale, such as the technology of DNA origami [37]. In its most basic definition, such self-assembling systems are: (i) comprised of parts or components that exhibit interaction; (ii) at thermodynamic nonequilibrium initially, but tend to equilibrium; (iii) thermodynamically closed [20,38]. Self-assembly is a process of energy minimization that ends in a final, well-defined structure that is uniquely determined by the properties of the interacting components which remain unchanged during the transition to thermodynamic equilibrium. Global order in the system is encoded in the initial set-up and the specific relationships that exist between components; no additional energy is necessary to drive the process [15].

An interesting example of self-assembly is found in the shaping of biological membranes, in particular lipid bilayers, that occur at the cellular level. Such objects are important building blocks which not only coat parts of the cell, such as the nucleus and the endoplasmic reticulum [40], but also form independent biological objects within the cell, such as vesicles and tubules which are necessary for the intra-cellular transport of wastes, nutrients, and proteins $[28,46]$.

The primary mechanism believed to be responsible for the highly curved geometries observed involves the recruitment of membrane-shaping proteins from the cellular fluid, such as the BAR (Bin/Amphiphysin Rvs) and ENTH (Epsin N-Terminal Homology) protein families [3, 9, 35, 51]. Such proteins bind directly onto the bilayer by means of electrostatic interactions and bend it by the insertion of amphipathic helix functional groups [13,30], with the magnitude of the induced curvature dependent on the depth of insertion into the lipid monolayer [21] and the number of attaching proteins [44]. Moreover, the BAR and ENTH families act as sensors of curvature [6,34], meaning that the shape of the membrane determines the adhesion kinetics of the proteins. In other words, we have a system where the curvature of the membrane regulates the concentration of attached proteins, and vice-versa, in an interacting process [41].

\footnotetext{
* Corresponding author

Email addresses: kwiecinski@maths.ox.ac.uk (James Kwiecinski), chapman@maths.ox.ac.uk (S. Jonathan Chapman), goriely@maths.ox.ac.uk (Alain Goriely)
} 
Previous investigations on the subject have been from two perspectives; namely, computational, involving large-scale coarse-grain simulations [2, 4, 29], and theoretical, which consider the minimization of membrane free energies or electrical potentials to determine the nature of equilibrium configurations. In the former, many interesting aspects of BAR proteins have been found, including the linear aggregation of proteins, leading to membrane tubulation [43, 49], and membrane fissioning, leading to closed tubular networks [42]. From a theoretical point of view, the effects of single BAR proteins, in the context of electric fields and potentials [25], and a continuum of them attaching to the lipid bilayer have also been studied, but not to the same extent as computational models. In particular, equilibrium configurations of the self-assembled system, and the role that BAR proteins play in the stability of these final shapes, are considered. Early research on the subject studied not the formation of vesicles or other independent biological objects, but rather the oscillations that can exist on the cell-membrane known as circular dorsal ruffles [24, 33]. It is found that BAR proteins provide a stabilization of the geometry; a point which has been further investigated in the context of flat membrane geometries [39] and pearling instabilities in cylindrical geometries [23].

As a starting point to understand this phenomenon, we derive the simplest, non-trivial system that allows us to explore the interplay between an underlying geometry and curvature-sensing proteins. We focus on the shaping of a filament and derive a macroscopic model for time-dependent self-assembly using concepts from continuum and statistical mechanics. The result is a thermodynamically consistent system of equations in terms of experimental parameters that allows us to further explore the role of the filament mechanics, the adhesion kinetics of the attaching proteins, and the interactions between these components.

\section{Mathematical Model}

Our mathematical model is based on the following assumptions: (i) We consider a thermodynamically closed system in 2D space which only contains the main continuum, the curvature-inducing proteins, and the interactions between these. There is no forcing or energy input from the outside environment; (ii) We take the continuum to be a 1D filament; explicitly, an elastic rod which is inextensible and unshearable with constant length $L$ that is parameterized with an arc-length coordinate $s \in[0, L]$. This geometry is a simplification of the 2D lipid bilayer without transverse mechanical effects and area dilation, despite the membrane being able to endure strains of 2-3\% [36]. The dependent variable of interest is the filament curvature $\kappa(t, s)$ at time $t$; (iii) The continuum freely floats in a fluid which is populated with a single type of BAR protein modeled as a thin filament with constant curvature. The proteins induce curvature along one principal direction which is always aligned with that of the main filament and they have a thermodynamically favorable target curvature $\kappa_{t}$. The number of bound proteins per unit length is given by $c(t, s)$.

Additionally, we make two important thermodynamic assumptions. (iv) We suppose that proteinmembrane interactions are at thermodynamic equilibrium with respect to energy exchange between these components. A number of theoretical models have made this same assumption [23, 33] as well as computational models [45]. In the latter case, results predicted by simulations have been experimentally verified [42], which suggests that this assumption is valid when studying qualitative aspects of the mathematical model.

(v) There are three stages in the protein attachment corresponding to the unbound, transition, and bound states. An unattached protein floating in the cellular fluid is assumed to have zero bending energy (unbound state), but when the protein attaches to the main filament, it deforms itself to match the curvature of the filament and acquires bending energy dictated by bending stiffness $B_{\mathrm{BAR}}$ (bound state). We suppose there is an intermediary step to attachment whereby the protein acquires bending energy determined by bending stiffness $B_{\mathrm{ts}}$, which is not necessarily the same as $B_{\mathrm{BAR}}$, and we assume that this transition state is sufficiently long-lived such that thermodynamic equilibrium is reached.

We now derive equations describing the time-evolution of $\kappa(t, s)$ and $c(t, s)$.

\subsection{Equations for Filamentary Mechanics}

We model the membrane cross-section as a Kirchhoff elastic filament whose motion is confined to a plane $[1,19]$. The position of the filament's centerline is denoted by $\mathbf{r}(t, s)$ and the curve is geometrically described by a local director basis $\left\{\mathbf{d}_{1}, \mathbf{d}_{2}, \mathbf{d}_{3}\right\}$ which is right-handed and orthonormal. The vectors $\mathbf{d}_{3}$ and $\mathbf{d}_{1}$ are the tangent and normal Frenet vectors respectively, whilst $\mathbf{d}_{2}$ points perpendicularly outwards from the plane and is constant with respect to both $t$ and $s$ (see Fig. 1). To complete the geometric description, we introduce 


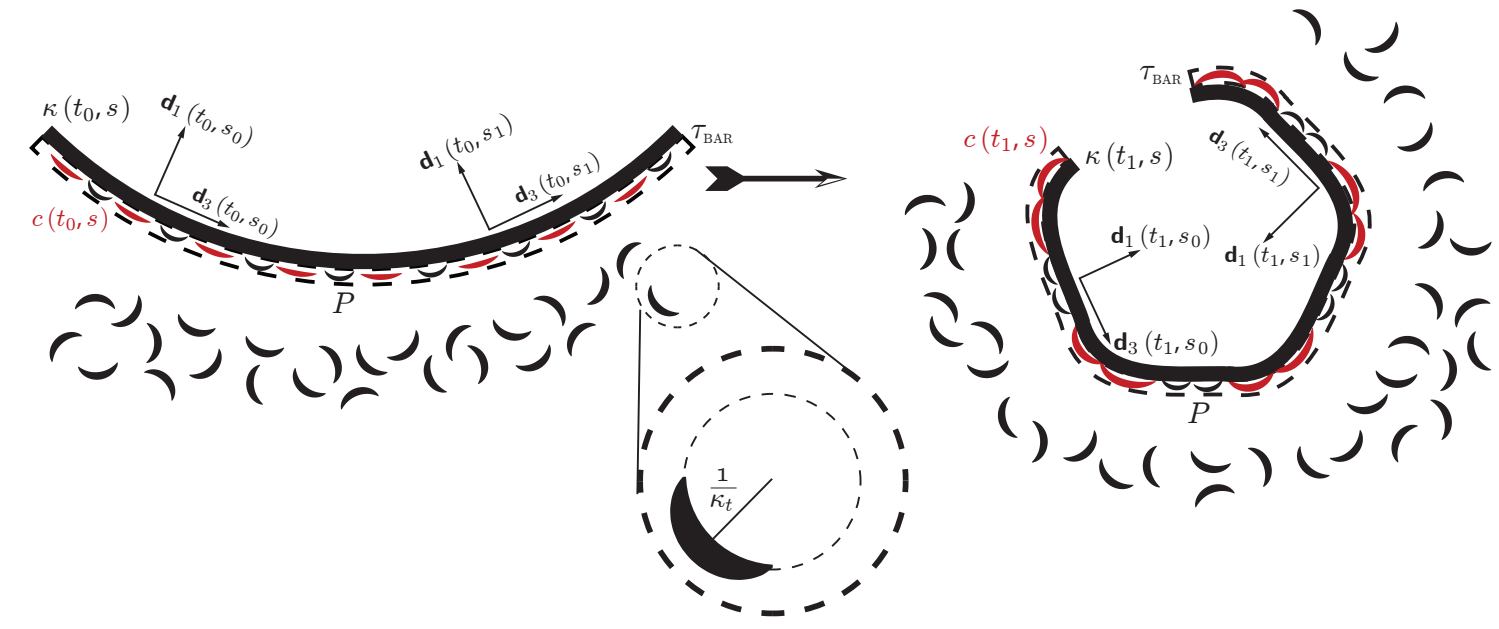

Figure 1: The mathematical model of $1 \mathrm{D}$ self-assembly as it evolves from $t=t_{0}$ to $t=t_{1}$. A filament of curvature $\kappa(t, s)$, that is described by local normal and tangential vectors $\mathbf{d}_{1}(t, s)$ and $\mathbf{d}_{3}(t, s)$, is submersed in a fluid populated with a single type of BAR protein which has constant intrinsic curvature $\kappa_{t}$. Unbound proteins in the layer of thickness $\tau_{\mathrm{BAR}}$ around the filament (shown as black) have constant concentration $P$, and can attach onto the filament resulting in a number per unit length $c(t, s)$ (shown as red). The attached proteins give the filament an intrinsic shape that evolves in time due to thermodynamic constraints on the protein adhesion kinetics. (Color online)

a strain vector $\mathbf{u}=\kappa \mathbf{d}_{2}$ and spin vector $\mathbf{w}=w_{2} \mathbf{d}_{2}$, with $w_{2}$ being a measure of the angular velocity of the director basis, so that we have the following kinematic relations:

$$
\begin{aligned}
\frac{\partial \mathbf{r}}{\partial s} & =\mathbf{d}_{3}, \\
\frac{\partial \mathbf{d}_{i}}{\partial s} & =\mathbf{u} \times \mathbf{d}_{i}, \\
\frac{\partial \mathbf{d}_{i}}{\partial t} & =\mathbf{w} \times \mathbf{d}_{i} .
\end{aligned}
$$

Defining the velocity of the rod in the local basis $\mathbf{v}=v_{1} \mathbf{d}_{1}+v_{3} \mathbf{d}_{3}=\partial \mathbf{r} / \partial t$, we use (1)-(3) to obtain geometric constraints for $v_{1}$ and $v_{3}$ :

$$
\begin{aligned}
0 & =\frac{\partial v_{1}}{\partial s}+\kappa v_{3}-w_{2} \\
0 & =\frac{\partial v_{3}}{\partial s}-\kappa v_{1}
\end{aligned}
$$

as well as a compatibility relation, given that $\partial^{2} \mathbf{d}_{i} / \partial s \partial t=\partial^{2} \mathbf{d}_{i} / \partial t \partial s$ :

$$
\frac{\partial \kappa}{\partial t}=\frac{\partial w_{2}}{\partial s} .
$$

Considering the mechanics of the continuum, we suppose that the only contribution to the applied force comes from fluid drag, which is proportional to the velocity of the filament $\mathbf{v}$. More specifically, the applied force per unit length is $\mathbf{f}=\mathbf{f}_{1} \mathbf{d}_{1}+\mathbf{f}_{3} \mathbf{d}_{3}=-\eta_{1} v_{1} \mathbf{d}_{1}-\eta_{3} v_{3} \mathbf{d}_{3}$, where $\eta_{1}$ and $\eta_{3}$ are the drag coefficients per unit length in the normal and tangential directions. Given that we are interested in motions that occur at low Reynolds numbers, it is reasonable to assume that the inertial terms can be neglected so that the resulting dynamics are first order in time [18]. Furthermore, we use slender body theory to simplify the applied force so that $\eta_{1}=2 \eta_{3}$, where $\eta_{3}=2 \pi \mu / A \ln (L / r)$ for a filament of length $L$, cross-sectional area $A$, and radius $r \ll L$ which is submersed in a fluid of dynamic viscosity $\mu$ [27]. Introducing the resultant force $\mathbf{n}=n_{1} \mathbf{d}_{1}+n_{3} \mathbf{d}_{3}$ and moment $\mathbf{m}$, we balance linear and angular momenta to obtain two equations of motion 
[32]:

$$
\begin{aligned}
\frac{\partial \mathbf{n}}{\partial s}+\mathbf{f} & =\rho A \frac{\partial^{2} \mathbf{r}}{\partial t^{2}}, \\
\frac{\partial \mathbf{m}}{\partial s}+\mathbf{d}_{3} \times \mathbf{n} & =\rho I\left(\mathbf{d}_{1} \times \frac{\partial^{2} \mathbf{d}_{1}}{\partial t^{2}}\right),
\end{aligned}
$$

for a filament of constant density $\rho$ and second moment of area $I$.

Lastly, to relate mechanical stresses to strains, we use a linear constitutive relation of the form:

$$
\mathbf{m}=E I(\kappa-\gamma c) \mathbf{d}_{2},
$$

where $E$ is the Young's modulus of the rod, the number of proteins per unit length $c$ takes on a role that is analogous to the intrinsic curvature, and $\gamma>0$ is a dimensionless measure of the curvature the proteins induce. The latter is included to account for the observation that the locally induced curvature is proportional to the number of proteins that have attached to the site [44] as well as the depth of amphipathic helix insertion [21].

Relation (9) is derived from an elastic energy which is quadratic in the curvature of the filament, however it has the same form as the Helfrich energy of a lipid bilayer reduced to a $1 \mathrm{D}$ geometry [22]. Furthermore, using (9) incurs an error $O\left(\omega^{2}\right)$ where [7]:

$$
\omega=\max _{s \in[0, L]}\{|\kappa(t, s)| r,|c(t, s)| r, r / L\} .
$$

Combining (1)-(3) with (7)-(9) and the aforementioned simplifications, we include (4)-(6) so that we have the following system of 6 equations for 6 dependent variables $\left\{v_{1}, v_{3}, w_{2}, \kappa, n_{1}, n_{3}\right\}$ :

$$
\begin{aligned}
\rho A \frac{\partial v_{1}}{\partial t} & =\frac{\partial n_{1}}{\partial s}+\kappa n_{3}-\rho A w_{2} v_{3}-\eta_{1} v_{1} \\
\rho A \frac{\partial v_{3}}{\partial t} & =\frac{\partial n_{3}}{\partial s}-\kappa n_{1}+\rho A w_{2} v_{1}-\frac{\eta_{1}}{2} v_{3} \\
\rho I \frac{\partial w_{2}}{\partial t} & =E I \frac{\partial}{\partial s}(\kappa-\gamma c)+n_{1} \\
\frac{\partial \kappa}{\partial t} & =\frac{\partial w_{2}}{\partial s} \\
0 & =\frac{\partial v_{1}}{\partial s}+\kappa v_{3}-w_{2} \\
0 & =\frac{\partial v_{3}}{\partial s}-\kappa v_{1}
\end{aligned}
$$

To close the system, we impose free-ends boundary conditions, which implies that $\mathbf{n}=\mathbf{m}=0$ at $s=$ $\{0, L\}$. In terms of the dependent variables, this corresponds to $n_{1}=n_{3}=0$ and $\kappa=\gamma c$ applying at both ends of the filament.

\subsection{Equation for Protein Adhesion Kinetics}

We suppose there are freely floating proteins in the cellular fluid which is taken to occupy an area much larger than that of the filament. The solution is assumed to be of sufficiently high concentration and wellmixed such that the proteins are uniformly distributed. We define a region of constant width around the filament that is one BAR protein thick $\tau_{\mathrm{BAR}}$ and presume that proteins can attach to the filament or detach from it in this region. The number of freely floating proteins per unit area of the region is $P$ whilst the number per unit length of proteins bound to the filament is $c$ (see Fig. 1). We summarize the kinetics as a chemical reaction of the form:

$$
P \underset{K_{r}(\kappa)}{\stackrel{K_{f}(\kappa) \tau_{\mathrm{BAR}}}{\rightleftharpoons}} c
$$

where $K_{f}(\kappa)$ and $K_{r}(\kappa)$ are the forward (attachment) and reverse (detachment) rates of reaction, both of which depend on the curvature of the filament. 
We apply the principle of mass action to (17) and write the time-evolution of $c$ as:

$$
\frac{\partial c}{\partial t}=K_{r}(\kappa)\left[\frac{K_{f}(\kappa)}{K_{r}(\kappa)} \tau_{\mathrm{BAR}} P-c\right] .
$$

If the assumptions regarding the cellular fluid hold in regards to its spatial uniformity and high concentration, and we additionally suppose that $\tau_{\mathrm{BAR}}$ is much smaller than the length of the filament $L$, then changes to $P$ will be instantly replenished by the surrounding solution. The latter assumption is biologically feasible given that $\tau_{\mathrm{BAR}} \lesssim 2 \times 10^{-9} \mathrm{~m}$, with the upper bound corresponding to the thickness of DNA [26], and $L \approx 10^{-7}$ from discussions on tube radii [5]. As such, $P$ is effectively constant and is treated as such in the forthcoming analysis.

To find explicit expressions for the rates of reaction, we use assumption (iv) and assign a Boltzmann factor to the ratio of reaction rates:

$$
\frac{K_{f}(\kappa)}{K_{r}(\kappa)}=\exp \left(-\frac{\Delta E}{k_{B} T}\right),
$$

where $k_{B}$ is Boltzmann's constant, $T$ is the absolute temperature in the system, and $\Delta E$ is the energy difference of the protein as it transitions from the unbound to the bound state.

Since the BAR protein is modeled as a thin filament of constant curvature, it has a microscopic bending energy given by [17]:

$$
\Delta E=E_{b}-E_{u}+\frac{B_{\mathrm{BAR}} L_{\mathrm{BAR}}}{2}\left(\kappa-\kappa_{t}\right)^{2},
$$

where $E_{u}$ and $E_{b}$ are the free energies of the protein in the unbound and bound state respectively, $B_{\mathrm{BAR}}$ is the bending stiffness of the protein in the bound state, $L_{\mathrm{BAR}}$ is the length of the protein, and $\kappa_{t}$ is the thermodynamically favorable target curvature that is equivalent to the intrinsic curvature of the protein.

Combining (19) and (20), we have:

$$
\frac{K_{f}(\kappa)}{K_{r}(\kappa)}=K_{\mathrm{eqm}} \exp \left(-\frac{\xi L_{\mathrm{BAR}}}{2}\left(\kappa-\kappa_{t}\right)^{2}\right),
$$

where $\xi=B_{\mathrm{BAR}} / k_{B} T>0$ is the persistence length of the protein, being a measure of its mechanical stiffness to thermal fluctuations, and $K_{\text {eqm }}=\exp \left(\left(E_{u}-E_{b}\right) / k_{B} T\right)>0$ is a quantity that measures the extent of the attachment reaction; that is, if $K_{\text {eqm }} \gg 1$, then most of the proteins will attach onto the filament, whilst if $K_{\text {eqm }} \ll 1$, very few will attach.

To determine expressions for $K_{f}(\kappa)$ and $K_{r}(\kappa)$, we use assumption (v) and introduce an intermediate state so that the kinetics of (17) now read:

$$
P \underset{K_{\text {int }}}{\stackrel{K_{f}(\kappa) \tau_{\mathrm{BAR}}}{\rightleftharpoons}} c_{\text {int }} \underset{K_{r}(\kappa)}{\stackrel{K_{\mathrm{int}}}{\rightleftharpoons}} c,
$$

where $K_{\text {int }}$ is the rate of the intermediate reaction and is assumed to be independent of $\kappa$, and $c_{\text {int }}$ is the number per unit length of proteins attached to the filament that are in the transition state.

Using (22), we can find $K_{f}(\kappa)$ by assigning a Boltzmann factor in a similar manner as before, with the energy difference now being between the unbound state and the transition state of the protein [11]:

$$
K_{f}(\kappa)=K_{f 0} K_{\mathrm{int}} \exp \left(-\frac{\xi_{\mathrm{ts}} L_{\mathrm{BAR}}}{2}\left(\kappa-\kappa_{t}\right)^{2}\right),
$$

where $\xi_{\mathrm{ts}}=B_{\mathrm{ts}} / k_{B} T>0, K_{f 0}=\exp \left(\left(E_{u}-E_{\mathrm{ts}}\right) / k_{b} T\right)$, and $E_{\mathrm{ts}}$ is the free energy of the protein in the transition state.

By considering the energy difference between the bound and the transition state, we find $K_{r}(\kappa)$ :

$$
K_{r}(\kappa)=K_{r 0} K_{\mathrm{int}} \exp \left(\frac{L_{\mathrm{BAR}}}{2}\left(\xi-\xi_{\mathrm{ts}}\right)\left(\kappa-\kappa_{t}\right)^{2}\right),
$$

where $K_{r 0}=\exp \left(\left(E_{b}-E_{\mathrm{ts}}\right) / k_{b} T\right)>0$ and is related to $K_{\text {eqm }}$ and $K_{f 0}$ by $K_{\text {eqm }}=K_{f 0} / K_{r 0}$. 
Some comments regarding (23) and (24) are necessary. We note the former predicts that the highest attachment rate occurs when the curvature of the filament $\kappa$ matches the target curvature of the protein $\kappa_{t}$. This result is expected given that the attaching protein does not need to bend to match its curvature with that of the filament, meaning no energy cost is incurred during the attachment process. Similar reasoning holds for (24), provided that $\xi>\xi_{\mathrm{ts}}$, which then implies that the detachment reaction for a filament curvature $\kappa \neq \kappa_{t}$ occurs at a higher rate than when $\kappa=\kappa_{t}$ (i.e. $K_{r}(\kappa)>K_{r}\left(\kappa_{t}\right)$ ). Proteins attaching to the filament for $\kappa \neq \kappa_{t}$ incur an energy cost and are thermodynamically undesirable, so they do not bind to the same extent as when $\kappa=\kappa_{t}$.

However, there is nothing to suggest that $\xi_{\text {ts }}>\xi$ cannot also occur. In this case, the opposite holds true; namely, if the filament curvature is $\kappa \neq \kappa_{t}$ then the protein will detach at a lower rate than when $\kappa=\kappa_{t}$. This is counter-intuitive given the previous reasoning, however this is a physically realizable system in the form of a simple toy: the Chinese finger trap. The idea of the toy is simple: it is easy to insert one's fingers into the trap, but it is difficult to pull them out using force. The reason is that when the fingers are forced out, stresses are created in the system that trap the fingers. In a similar manner, stresses that are generated by the mismatch of curvatures between the filament and the protein cause the attached protein to be trapped. In either case, this mathematical model is able to emulate both physically realizable scenarios.

Lastly, we suppose that proteins that have attached onto the filament can move in two ways: the diffusion of proteins from regions of high concentration to low concentration and the drifting of proteins which seek to minimize the quantity $\left(\kappa-\kappa_{t}\right)^{2}$ for the thermodynamic reasons outlined previously. Combining the effects of protein movement with (18), (21), and (24), we obtain:

$$
\begin{aligned}
\frac{\partial c}{\partial t}=K_{r 0} K_{\mathrm{int}} \exp \left(\frac{L_{\mathrm{BAR}}}{2}\left(\xi-\xi_{\mathrm{ts}}\right)\left(\kappa-\kappa_{t}\right)^{2}\right)\left\{K_{\mathrm{eqm}} \tau_{\mathrm{BAR}} P \exp (\right. & \left.\left.-\frac{\xi L_{\mathrm{BAR}}}{2}\left(\kappa-\kappa_{t}\right)^{2}\right)-c\right\} \\
& +D \frac{\partial^{2} c}{\partial s^{2}}+\chi \frac{\partial}{\partial s}\left[c \frac{\partial}{\partial s}\left(\kappa-\kappa_{t}\right)^{2}\right],
\end{aligned}
$$

where $D>0$ is the diffusion constant of proteins on the filament and $\chi>0$ is the sensitivity of an individual protein to move towards regions with low $\left(\kappa-\kappa_{t}\right)^{2}$. The drift term shares a similar form to the NernstPlanck equation for charged particles minimizing their electric potential [50] and the Keller-Segel model for the movement of bacterial populations due to gradients in chemoattractants [47].

To close (25), we suppose that no attached proteins flow off the edges of the filament, so that $\partial c / \partial s=0$ at $s=\{0, L\}$.

\subsection{D Self-Assembly Equations}

Equations (11)-(16) and (25) form the 1D self-assembly system. We introduce the following nondimensionalizations and rescaling to simplify: $\hat{s}=s / L, \hat{t}=E I t / \eta_{1} L^{4}, \hat{\kappa}=\kappa L, \hat{c}=\gamma c L, \hat{n}_{1}=n_{1} L^{2} / E I$, $\hat{n}_{3}=n_{3} L^{2} / E I, \hat{v}_{1}=v_{1} \eta_{1} L^{3} / E I, \hat{v}_{3}=v_{3} \eta_{1} L^{3} / E I, \hat{w}_{2}=w_{2} \eta_{1} L^{4} / E I, \hat{\kappa}_{t}=\kappa_{t} L, \hat{P}=\gamma K_{\mathrm{eqm}} \tau_{\mathrm{BAR}} P L$, $\hat{\xi}=\xi L_{\mathrm{BAR}} / L^{2}, \hat{\xi}_{\mathrm{ts}}=\xi_{\mathrm{ts}} L_{\mathrm{BAR}} / L^{2}, \hat{K}_{r 0}=K_{r 0} K_{\mathrm{int}} \eta_{1} L^{4} / E I, \hat{D}=D \eta_{1} L^{2} / E I, \hat{\chi}=\chi \eta_{1} / E I$, so that the equations of interest become (upon dropping the hat symbol):

$$
\begin{aligned}
\alpha \frac{\partial v_{1}}{\partial t} & =\frac{\partial n_{1}}{\partial s}+\kappa n_{3}-\alpha w_{2} v_{3}-v_{1} \\
\alpha \frac{\partial v_{3}}{\partial t} & =\frac{\partial n_{3}}{\partial s}-\kappa n_{1}+\alpha w_{2} v_{1}-\frac{v_{3}}{2} \\
\beta \frac{\partial w_{2}}{\partial t} & =\frac{\partial}{\partial s}(\kappa-c)+n_{1} \\
\frac{\partial \kappa}{\partial t} & =\frac{\partial w_{2}}{\partial s} \\
0 & =\frac{\partial v_{1}}{\partial s}+\kappa v_{3}-w_{2}, \\
0 & =\frac{\partial v_{3}}{\partial s}-\kappa v_{1},
\end{aligned}
$$




\begin{tabular}{|c|c|}
\hline Quantity & Estimated Value \\
\hline \hline$\kappa, \kappa_{t}, c, P$ & $1-10$ \\
\hline$\xi, \xi_{\text {ts }}$ & $10^{-3}-10$ \\
\hline$K_{r 0}$ & $10^{-12}-10^{12}$ \\
\hline$D, \chi$ & $10^{10}-10^{12}$ \\
\hline$\alpha, \beta$ & $10^{-52}-10^{-36}$ \\
\hline
\end{tabular}

Table 1: Estimates of important variables and quantities that appear in (26)-(32).

where $\alpha=\rho A E I / \eta_{1}^{2} L^{4} \sim r^{10} / L^{4}$ and $\beta=\rho E I^{2} / \eta_{1}^{2} L^{6} \sim r^{12} / L^{6}$, both of which are small given that $r \ll L$ as seen in Section 2.1, and

$$
\frac{\partial c}{\partial t}=K_{r 0} \exp \left(\frac{1}{2}\left(\xi-\xi_{\mathrm{ts}}\right)\left(\kappa-\kappa_{t}\right)^{2}\right)\left\{P \exp \left(-\frac{\xi}{2}\left(\kappa-\kappa_{t}\right)^{2}\right)-c\right\}+D \frac{\partial^{2} c}{\partial s^{2}}+\chi \frac{\partial}{\partial s}\left[c \frac{\partial}{\partial s}\left(\kappa-\kappa_{t}\right)^{2}\right],
$$

with boundary conditions given by $n_{1}=n_{3}=0, \kappa=c$, and $\partial c / \partial s=0$ at $s=\{0,1\}$.

For the current analysis and following numerical studies, we include estimates of some of the important quantities for a typical biological system in Table 1. In particular, we have used estimates for curvature based on discussions in [5], which propose the radius of remodeled tubes to be of the order $10^{-8}-10^{-7} \mathrm{~m}$, and have assumed that the length of the filament is such that the self-assembled system forms complete constant curvature solutions with little to no overlap. We estimate $\eta_{1} \approx 10^{13}-10^{14}$, taking the dynamic viscosity of fluid to be approximately that of water $\mu \approx 10^{-4} \mathrm{~m}^{2} \mathrm{~s}^{-1}$ and the radius of the filament $r \approx 10^{-9} \mathrm{~m}$ to coincide with the thickness of a lipid bilayer. We consider the bilayer to be made of packed hydrocarbon chains and use a Young's modulus of $E \approx 10^{7}-10^{8} \mathrm{Nm}^{-2}$, which provides a reasonably good estimate of the material properties of a biological membrane according to [12], and take the density of the filament to be $\rho \approx 10^{3} \mathrm{~kg} \cdot \mathrm{m}^{-3}$ to approximate the fluid nature of the bilayer. Furthermore, we estimate the protein persistence length to be $\xi \approx 10^{-12}-10^{-8} \mathrm{~m}$, with the larger bound coinciding with that of DNA [31], and the diffusion of proteins on the filament to be $D \approx 10^{-12}-10^{-11} \mathrm{~m}^{2} \mathrm{~s}^{-1}$, according to studies of membrane protein movement on lipid bilayers found in [48]. Lastly, we assume $K_{r 0}$ varies substantially based upon chemical triggers which can affect protein adhesion.

Given the small estimated values of $\alpha$ and $\beta$, we consider the limit $\alpha, \beta \rightarrow 0^{+}$in the forthcoming analysis.

\section{Stability Analysis of Constant Curvature Solutions}

\subsection{Steady States}

To determine the time-independent solutions of (26)-(32), we first consider when such solutions occur in the mechanics of the filament. We denote the steady state of a variable by ()$^{\star}$. Given that $v_{1}^{\star}=v_{3}^{\star}=0$, (7) reduces to $\partial \mathbf{n}^{\star} / \partial s=\mathbf{0}$ since the only applied force comes from fluid drag, which implies that $\mathbf{n}^{\star}=\mathbf{0}$ over the entire filament from the free-ends boundary conditions. Similarly from (8), $\partial \mathbf{m}^{\star} / \partial s=\mathbf{0}$, which implies $\mathbf{m}^{\star}=\mathbf{0}$ for all $s$ using the same reasoning. In terms of dependent variables, steady states occur when $v_{1}^{\star}=v_{3}^{\star}=w_{2}^{\star}=n_{1}^{\star}=n_{3}^{\star}=0$ and $\kappa^{\star}=c^{\star}$ throughout the filament.

However, the adhesion kinetics of the proteins specifies that only certain solutions for $c^{\star}$ will be allowed due to thermodynamic constraints, the form of which are determined by solving (32) for $\partial c^{\star} / \partial t=0$. For the present analysis however, we focus on steady solutions which are spatially uniform. This simplification gives the following criterion for time-independent states using the result $\kappa^{\star}=c^{\star}$ :

$$
f\left(\kappa^{\star}\right)=P \exp \left(-\frac{\xi}{2}\left(\kappa^{\star}-\kappa_{t}\right)^{2}\right)-\kappa^{\star}=0,
$$

which can have 1,2 , or 3 solutions all of which are non-zero and positive, given that $P>0$. This latter point is illustrated by plotting $f\left(\kappa^{\star}\right)$ for the parameters $P=10$ and $\kappa_{t}=5.5$ with (a) $\xi=0.05$, (b) $\xi=0.2274$, and (c) $\xi=0.8$ in Fig. 2. It is interesting to note that the possibility of up to 3 constant curvature steady states agrees with previous work which was based on minimizing the free-energy associated with a cylindrical lipid bilayer tube [23]. 
We note that if $\xi \gg 1$, corresponding to low temperature environments or proteins with large bending stiffness, then (33) suggests that $\kappa^{\star} \rightarrow\left\{0^{+}, \kappa_{t}^{-}, \kappa_{t}^{+}\right\}$depending on the extent of protein attachment and the concentration of proteins in the surrounding region, both of which are encoded in $P$. If $P$ is sufficiently large, then proteins can be thermodynamically forced to attach and, given that the bending stiffness of the proteins is very large, the curvature of the filament is largely determined by the intrinsic curvature of the protein which attaches and acts as a scaffold when uniformly distributed along the rod, leading to $\kappa^{\star} \rightarrow\left\{\kappa_{t}^{-}, \kappa_{t}^{+}\right\}$. If $P$ is not sufficiently large, then it is thermodynamically undesirable for any proteins to attach to the filament given the large amount of energy necessary to bend the proteins so that they match the filament shape, so $\kappa^{\star} \rightarrow 0^{+}$.

\subsection{Linear Stability Analysis}

We now study the stability of the spatially uniform steady states subject to imposed perturbations. We linearize around the equilibrium solutions of (26)-(32) for $\alpha, \beta \rightarrow 0^{+}$by introducing the ansatz $h=h^{\star}+$ $\delta_{h} \exp (i k s+\sigma t)$ for $h=\left\{v_{1}, v_{3}, w_{2}, \kappa, n_{1}, n_{3}, c\right\}$, where $h^{\star}$ satisfies the time-independent constant curvature solutions discussed in Section 3.1 and it is assumed that $\delta_{h} \ll 1$. The real part of the parameter $\sigma$ determines whether a perturbation with wavenumber $k$ will grow or decay in time. The goal is to find a dispersion relation connecting $\sigma$ and $k$.

We first apply the ansatz to the mechanics of the filament (i.e. (26)-(31)) and find that all $\delta_{j}$ for $j=\left\{v_{1}, v_{3}, w_{2}, n_{1}, n_{3}\right\}$ can be expressed in terms of $\delta_{\kappa}$ and $\delta_{c}$ to linear order. Eliminating these $\delta_{j}$ gives the following relation between $\delta_{\kappa}$ and $\delta_{c}$ :

$$
\sigma \delta_{\kappa}=H\left(\delta_{c}-\delta_{\kappa}\right)
$$

where $H=2 k^{2}\left(k^{2}-\kappa^{\star 2}\right)^{2} /\left(2 k^{2}+\kappa^{\star 2}\right)$.

Applying the ansatz to protein adhesion (i.e. (32)), we can use Taylor expansions around $\delta_{\kappa}=0$ to approximate the exponentials and neglect $\delta_{\kappa} \delta_{c}$ and $\delta_{k}^{2}$ terms to derive the relation:

$$
\sigma \delta_{c}=F \delta_{\kappa}-G \delta_{c}
$$

where:

$$
\begin{aligned}
F & =A B \xi P\left(\kappa_{t}-\kappa^{\star}\right)-2 \chi \kappa^{\star}\left(\kappa^{\star}-\kappa_{t}\right) k^{2}, \\
G & =A+D k^{2} \\
A & =K_{r 0} \exp \left(\frac{1}{2}\left(\xi-\xi_{\mathrm{ts}}\right)\left(\kappa^{\star}-\kappa_{t}\right)^{2}\right)>0, \\
B & =\exp \left(-\frac{\xi}{2}\left(\kappa^{\star}-\kappa_{t}\right)^{2}\right)>0 .
\end{aligned}
$$

We express (34)-(35) in matrix form:

$$
\sigma\left[\begin{array}{l}
\delta_{\kappa} \\
\delta_{c}
\end{array}\right]=\left[\begin{array}{cc}
-H & H \\
F & -G
\end{array}\right]\left[\begin{array}{l}
\delta_{\kappa} \\
\delta_{c}
\end{array}\right]=M\left[\begin{array}{c}
\delta_{\kappa} \\
\delta_{c}
\end{array}\right]
$$

so that $\sigma$ is an eigenvalue of $M$. The characteristic polynomial for $\sigma$ is:

$$
0=\sigma^{2}+(G+H) \sigma+H(G-F),
$$

which has real solutions except if the system is dominated by protein drift (i.e. $\chi \gg D, K_{r 0}$ ).

This is the dispersion relation which determines $\sigma$ as a function of $k$. In the short wave limit (i.e. $k \rightarrow \infty$ ), we have:

$$
\begin{aligned}
\sigma_{1} & =-k^{4}+O\left(k^{2}\right), \\
\sigma_{2} & =-D_{\text {eff }} k^{2}+O(1),
\end{aligned}
$$

whilst in the long wave limit (i.e. $k \rightarrow 0^{+}$):

$$
\begin{aligned}
\sigma_{1} & =\frac{2 \kappa^{\star 2}}{A}\left(F_{0}-A\right) k^{2}+O\left(k^{4}\right), \\
\sigma_{2} & =-A+O\left(k^{2}\right),
\end{aligned}
$$


where $D_{\text {eff }}=D+2 \chi \kappa^{\star}\left(\kappa^{\star}-\kappa_{t}\right)$ is the effective diffusion of proteins on the filament with the inclusion of drift effects and $F_{0}=\lim _{\chi \rightarrow 0} F$.

Some comments regarding (42)-(45) are in order. First, (42) and (43) suggest that $\sigma \leq 0$ for $k \rightarrow \infty$, provided $D_{\text {eff }} \geq 0$, which implies that the $1 \mathrm{D}$ self-assembly system is well-posed. However, since $D, \chi, \kappa^{\star}>0$, $D_{\text {eff }}$ can become negative when the curvature of the filament is less than the target curvature of the protein $\kappa^{\star}<\kappa_{t}$ and protein movement is drift dominated, $\chi \gg D$. For the forthcoming analysis, we only consider well-posed systems where $D>\chi$.

Second, (44) shows that the stability of the time-independent constant curvature solutions is determined by $F_{0}-A$, which only depends on the shape of the filament and the nature of the protein adhesion kinetics. The process of protein attachment can both stabilize and destabilize the curvature of the filament. Instabilities arise when $F_{0}-A>0$, provided $D_{\text {eff }}>0$, which reduces to the criterion $\kappa_{t}-\kappa^{\star}>1 / B \xi P$. Given that $B, \xi, P>0$, unstable solutions only exist for filament curvatures less than the target protein curvature $\kappa^{\star}<\kappa_{t}$. Additionally, the magnitude of the instability is directly proportional to the degree of protein recruitment $K_{r 0}$ and the extent of protein attachment to the filament together with the concentration of proteins in the surrounding region $P$.

To summarize, we generate a bifurcation diagram of the constant curvature steady states as a function of $\xi$ for $P=10$ and $\kappa_{t}=5.5$ in Fig. 2. We note that upon initially increasing the persistence length from 0 , there is only one spatially uniform and globally stable solution until $\xi=\xi_{\text {crit }} \approx 0.2274$, such that $F_{0}-A=0$. A saddle-node bifurcation appears which splits into two other possible solutions, one being linearly stable and of low curvature and the other being linearly unstable. As $\xi$ increases even further, the unstable and highly curved stable solution asymptotically approach $\kappa_{t}=5.5$ as $\xi \rightarrow \infty$, given the thermodynamic forcing from $P$.

We also create phase diagrams of $P / \kappa_{t}$ vs. $\xi \kappa_{t}^{2}$ to show the linearly stable and unstable regions for $\kappa^{\star}=\left\{2.6996 \times 10^{-6}, 4.1790,6.4384\right\}$ (shown in Fig. 3(a), (b), and (c) respectively) with $\kappa_{t}=5.5$ in the regime of biologically realistic parameters, according to Table 1 . We observe that when increasing $\xi / \kappa_{t}^{2}$ from 0 whilst keeping $\kappa^{\star}$ constant, the low and high curvature solutions remain linearly stable whilst the middle solution transitions from stable to unstable at a critical value of $\xi_{\text {crit }} \kappa_{t}^{2} \approx 5.4796$. When $\xi \kappa_{t}^{2}>\xi_{\text {crit }} \kappa_{t}^{2}$, the system features a bistable regime which is divided by the linearly unstable middle curvature solution.

\subsection{Weakly Nonlinear Analysis for $k \rightarrow 0^{+}$}

For $\xi>\xi_{\text {crit }}$, the self-assembly system has bistable high and low curvature solutions that are separated by an unstable branch. To determine the form of the resulting solution when the unstable curvature is perturbed, we study the immediate region around the bifurcation by introducing $F_{0}-A=\epsilon$ for $\epsilon \ll 1$. In this case, the nonlinearities are weak and the unstable modes grow slowly such that a functional form for the solution can be found by rescaling time and space [8].

At the bifurcation, $\sigma=0$ which suggests $\delta_{\kappa}=\delta_{c}$ and $\delta_{v_{1}}=\delta_{v_{3}}=\delta_{w_{2}}=\delta_{n_{1}}=\delta_{n_{3}}=0$ for $k \rightarrow 0^{+}$from the linear analysis. By using balancing arguments, we find the correct scaling is:

$$
\begin{aligned}
\kappa & =\kappa^{\star}+\epsilon \delta^{(0)}\left(t_{1}, s_{1}\right)+\epsilon^{2} \delta_{\kappa}^{(1)}\left(t_{1}, s_{1}\right)+O\left(\epsilon^{3}\right), \\
c & =\kappa^{\star}+\epsilon \delta^{(0)}\left(t_{1}, s_{1}\right)+\epsilon^{2} \delta_{c}^{(1)}\left(t_{1}, s_{1}\right)+O\left(\epsilon^{3}\right), \\
v_{1} & =\epsilon^{3} \delta_{v_{1}}^{(1)}\left(t_{1}, s_{1}\right)+O\left(\epsilon^{4}\right), \\
v_{3} & =\epsilon^{5 / 2} \delta_{v_{3}}^{(1)}\left(t_{1}, s_{1}\right)+O\left(\epsilon^{7 / 2}\right), \\
w_{2} & =\epsilon^{5 / 2} \delta_{w_{2}}^{(1)}\left(t_{1}, s_{1}\right)+O\left(\epsilon^{7 / 2}\right), \\
n_{1} & =\epsilon^{5 / 2} \delta_{n_{1}}^{(1)}\left(t_{1}, s_{1}\right)+O\left(\epsilon^{7 / 2}\right), \\
n_{3} & =\epsilon^{3} \delta_{n_{3}}^{(1)}\left(t_{1}, s_{1}\right)+O\left(\epsilon^{4}\right),
\end{aligned}
$$

for slow time scale $t_{1}=\epsilon^{2} t$ and rescaled space $s_{1}=\epsilon^{1 / 2} s$.

We divide the system into the contributions of the filament mechanics and the protein adhesion kinetics and consider each part separately. As before, we can express $\delta^{(0)}, \delta_{\kappa}^{(1)}$, and $\delta_{c}^{(1)}$ in terms of $\delta_{j}^{(1)}$ for $j=$ 


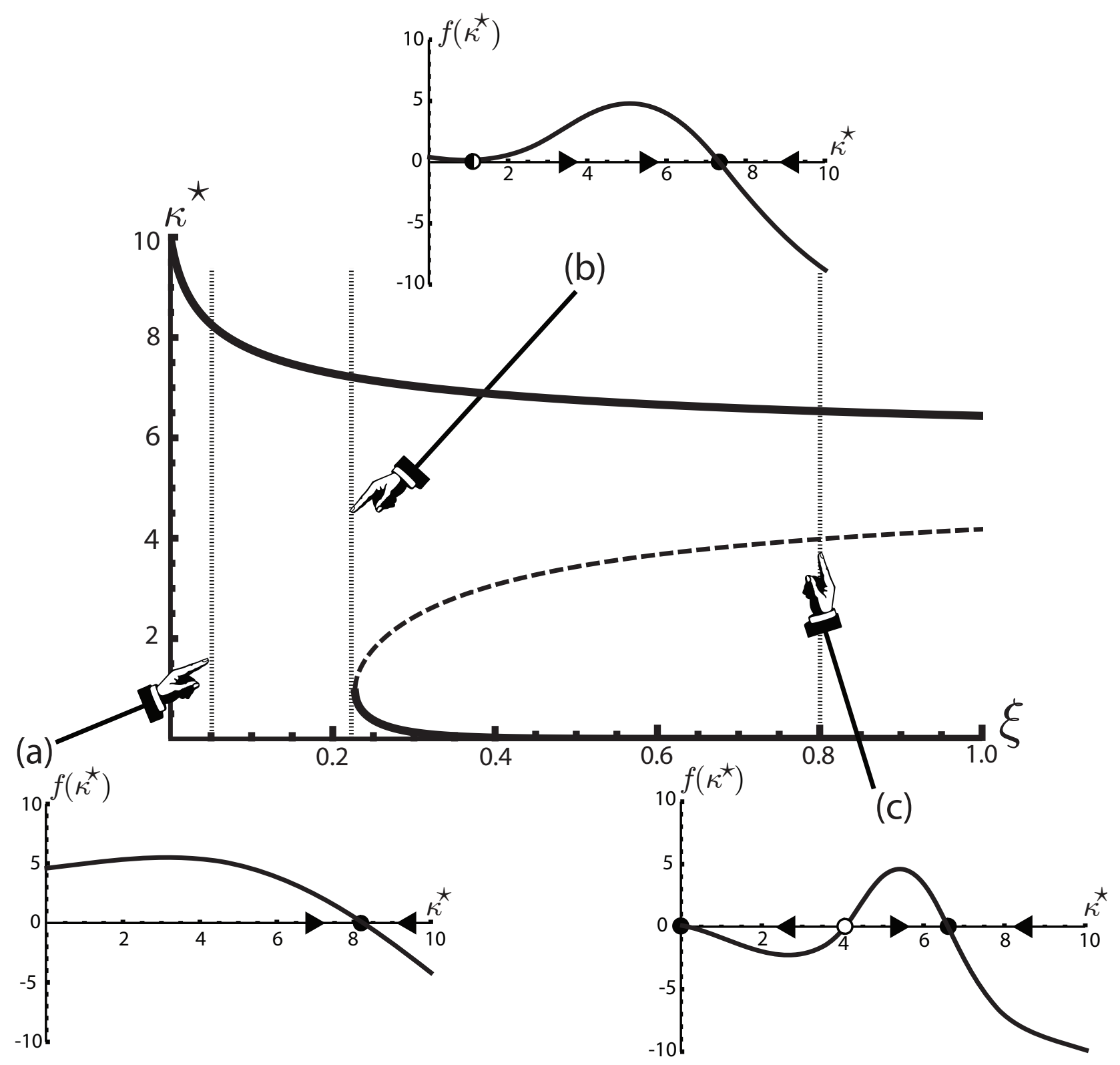

Figure 2: Bifurcation diagram of the spatially uniform steady states $\kappa^{\star}$ as a function of the protein persistence length $\xi$ and plots of $f\left(\kappa^{\star}\right)$, as defined in (33), with (a) $\xi=0.05<\xi_{\text {crit }}$, (b) $\xi=0.2274 \approx \xi_{\text {crit }}$, and (c) $\xi=0.8>\xi_{\text {crit }}$. The other parameters are $P=10$ and $\kappa_{t}=5.5$. As $\xi$ increases, there is one globally stable solution (denoted by a solid line) until $\xi=\xi_{\text {crit }} \approx 0.2274$, whereby a saddle-node bifurcation appears and introduces two new solutions; one which is linearly stable whilst the other is unstable (denoted by a dashed line). 
(a)

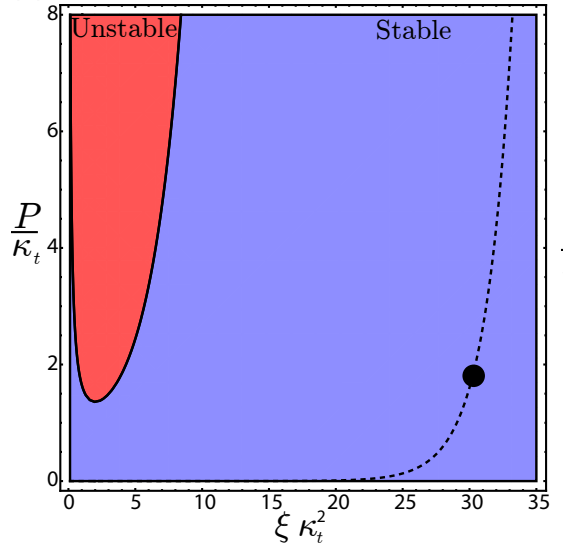

(b)

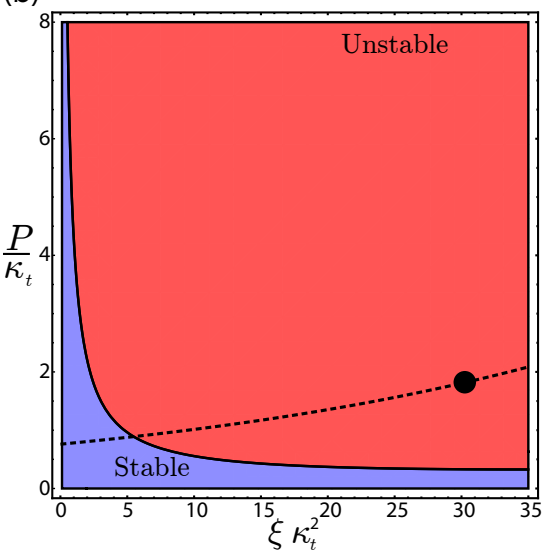

(c)

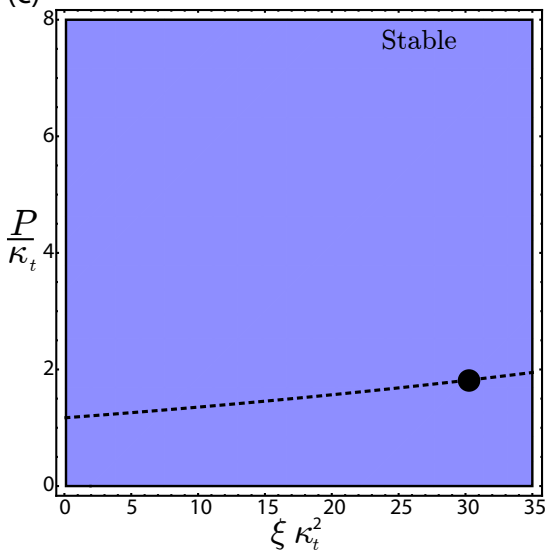

Figure 3: Phase diagrams of $P / \kappa_{t}$ vs. $\xi \kappa_{t}^{2}$ for $\kappa_{t}=5.5$ with (a) $\kappa^{\star}=2.6996 \times 10^{-6}$, (b) $\kappa^{\star}=4.1790$, and (c) $\kappa^{\star}=6.4384$. Blue and red zones correspond to linearly stable and unstable regions respectively, according to (44). $\kappa^{\star}$ is constant along the dashed line whilst the black dot corresponds to $\xi=1$ and $P=10$ used to generate Fig. 5 and 6. (Color online)

$\left\{v_{1}, v_{3}, w_{2}, n_{1}, n_{3}\right\}$ and find the contribution from the filament mechanics (i.e. (26)-(31)) reduces to:

$$
\frac{\partial \delta^{(0)}}{\partial t_{1}}=2 \kappa^{\star 2} \frac{\partial^{2}}{\partial s_{1}}\left(\delta_{\kappa}^{(1)}-\delta_{c}^{(1)}\right) .
$$

Applying a similar treatment to (32), we use Taylor expansions around $\epsilon=0$ to obtain:

$$
\delta_{\kappa}^{(1)}-\delta_{c}^{(1)}=-\frac{1}{A}\left(\delta^{(0)}-\nu\left(\delta^{(0)}\right)^{2}+D_{\mathrm{eff}} \frac{\partial^{2} \delta^{(0)}}{\partial s_{1}^{2}}\right)
$$

where $\nu=A B \xi P / 2-\left(\xi-\xi_{\mathrm{ts}}\right)\left(\kappa^{\star}-\kappa_{t}\right)\left(F_{0}-A\right) \geq 0$, given that $\kappa_{t}>\kappa^{\star}, F_{0}-A=\epsilon>0$ and the estimated values of $\xi, \xi_{\mathrm{ts}} \leq 10$.

We combine (53) and (54) to obtain a reduced equation that describes the time-evolution of long-wave perturbations to the unstable constant curvature solutions of $\kappa$ and $c$. We introduce the rescaling $\hat{\delta}^{(0)}=\nu \delta^{(0)}$, $\hat{s}_{1}=s_{1} / L_{1}, \hat{t}_{1}=2 D_{\text {eff }} \kappa^{\star 2} t_{1} / L_{1}^{4} A$, and $\hat{L}_{1}=L_{1} / D_{\text {eff }}^{1 / 2}$, where $L_{1}=\epsilon^{1 / 2}$ is the rescaled length of the filament, so that the equation becomes (upon dropping the hat symbol):

$$
\begin{aligned}
\mu & =-L_{1}^{2} \delta^{(0)}+L_{1}^{2}\left(\delta^{(0)}\right)^{2}-\frac{\partial^{2} \delta^{(0)}}{\partial s_{1}^{2}}, \\
\frac{\partial \delta^{(0)}}{\partial t_{1}} & =\frac{\partial^{2} \mu}{\partial s_{1}}
\end{aligned}
$$

with boundary conditions given by $\partial \delta^{(0)} / \partial s_{1}=0$, from $\partial c / \partial s=0$, and $\mu=0$, from $\kappa=c$ using (54), applicable at $s_{1}=\{0,1\}$.

Equations (55)-(56) form a Cahn-Hilliard type system which is the prototypical model for phase-separation between multiple stable states and subsequent phase coarsening, whereby the areas of a particular state diffuse and merge together forming larger and more homogeneous regions as time evolves. Such systems can be derived from a global energy functional [16] which, for the current system, is written as:

$$
\varepsilon=\int_{0}^{1} \frac{L_{1}^{2}}{3}\left(\delta^{(0)}\right)^{3}-\frac{L_{1}^{2}}{2}\left(\delta^{(0)}\right)^{2}+\frac{1}{2}\left(\frac{\partial \delta^{(0)}}{\partial s_{1}}\right)^{2} \mathrm{~d} s_{1} .
$$

Note, however, that unlike the usual Cahn-Hilliard system, this energy is not bounded below. This functional density has a cubic potential with an unstable state $\delta^{(0)}=0$ and two stable states $\delta^{(0)}=\{-\infty, 1\}$. 
Using the boundary conditions on $\delta^{(0)}$ and $\mu$, we have:

$$
\frac{\mathrm{d} \varepsilon}{\mathrm{d} t_{1}}=-\int_{0}^{1}\left(\frac{\partial \mu}{\partial s_{1}}\right)^{2} \mathrm{~d} s_{1} \leq 0,
$$

so that the energy is decreasing for all time. Defining the mass as $\phi=\int_{0}^{1} \delta^{(0)} \mathrm{d} s_{1}$, we find that:

$$
\frac{\mathrm{d} \phi}{\mathrm{d} t_{1}}=\left[\frac{\partial \mu}{\partial s_{1}}\right]_{0}^{1} \neq 0
$$

which implies that mass is not conserved, unlike the standard Cahn-Hilliard system, due to the given boundary conditions on $\mu$. Note that $\phi$ is physically related to the mass of attached proteins on the filament. However, given that the number of attached proteins can change due to the thermodynamic constraints on protein recruitment, it is not necessary that $\phi$ is conserved.

Result (59), coupled with the fact that the system energy decreases (i.e. (58)) and that $\delta^{(0)}=-\infty$ is a stable solution, might seem alarming in that it could result in unbounded solutions to (26)-(32) in the bistable regime. However, this aspect would not appear in the full self-assembly system because of the asymmetry that is built into the protein recruitment term derived in (18); namely, that $c \in[0, P]$. The concentration of attached proteins cannot be negative. Given that $c$ is bounded from below and that the perturbations to $c$ and $\kappa$ are the same to $O(\epsilon)$ in the long-wave regime, the curvature of the filament is finite too. In short, ansatzes (46)-(52) cease to be valid as the solution of the full self-assembly system reaches equilibrium far from the uniform unstable state.

The time-evolution of perturbations to the unstable curvature can be described in the framework of the Cahn-Hilliard system: For a given initial condition $\delta^{(0)}(0, s)$, the long wave perturbations will locally converge to the states $\delta^{(0)}=\{-\infty, 1\}$ forming patterns. These patterns will interact and merge to form more homogeneous regions of a particular stable state until the solution for $\delta^{(0)}$ is spatially uniform for all $s_{1}$. It should be noted that the phase-coarsening occurs on a time-scale that depends on the factor $A L_{1}^{4} / 2 D_{\text {eff }} \kappa^{\star 2}$, which means that for smaller effective protein movement $D_{\text {eff }}$, the resolution to a constant solution takes considerably longer. For instance, if $D_{\text {eff }}$ is vanishingly small, the patterns locally converge to the bistable solutions and uniformly resolve as $t \rightarrow \infty$. Diffusion and drift compete to smooth and preserve the pattern formation respectively for $\kappa^{\star}<\kappa_{t}$, given that $D_{\text {eff }}=D+2 \chi \kappa^{\star}\left(\kappa^{\star}-\kappa_{t}\right)$ increases for increasing $D$ and decreasing $\chi$.

If $\delta^{(0)}\left(t_{1}=0, s_{1}\right)=0$ is perturbed by a constant offset, we would expect that it resolves in a spatially uniform manner, however $L_{1}$ plays a role in (55)-(56). To illustrate this length dependence, we numerically solve (55)-(56), using a second order time-differencing method incorporating Newton-Raphson iteration, with initial condition $\delta^{(0)}(0, s)=10^{-9}$ for $L_{1}=2 \pi, L_{1}=4 \pi$, and $L_{1}=6 \pi$ (shown in Fig. 4(a), (b), and (c) respectively), which corresponds to decreasing $D_{\text {eff }}$ in the full system. For small $L_{1}, \delta^{(0)}$ converges to the spatially uniform $\delta^{(0)}=1$ solution, however as $L_{1}$ increases, the resolution is spatially inhomogeneous and is driven by the boundary terms, with the initial pattern formation becoming more oscillatory and occurring in shorter times for increasing $L_{1}$. Indeed, if we consider the condition $\mu=0$, then as $L_{1}$ is made larger and $\delta^{(0)} \neq 0$, the only way in which the boundary condition can be satisfied is if $\partial^{2} \delta^{(0)} / \partial s_{1}^{2}$ balances $-L_{1}^{2} \delta^{(0)}+L_{1}^{2}\left(\delta^{(0)}\right)^{2}$. In other words, a non-zero second order derivative is introduced at the boundary, the magnitude of which is directly related to $L_{1}$, and drives the dynamics of the system so that the solution locally converges to $\delta^{(0)}=\{-\infty, 1\}$.

\subsection{Pattern Formation and Phase-Coarsening in Protein Concentration}

To verify the results predicted by system (55)-(56), we numerically solve (26)-(32), using a first order implicit time-differencing method and second-order spatial finite differencing, for $\kappa$ in the region of the linearly unstable solution shown in Fig. 2. The numerical scheme is as follows:

(i) We start the system with an initial condition that satisfies the steady state of the filament, but not the adhesion kinetics of the protein; that is, $\kappa^{\dagger}=c^{\dagger}, n_{1}^{\dagger}=n_{3}^{\dagger}=v_{1}^{\dagger}=v_{3}^{\dagger}=w_{2}^{\dagger}=0$, but $\kappa^{\dagger}$ does not satisfy (33). This requirement bypasses the need for the shooting method often necessary for boundary value problems relating to the Kirchhoff rod, given that all dependent variables associated with the mechanics are known throughout the filament a priori. 
(a)

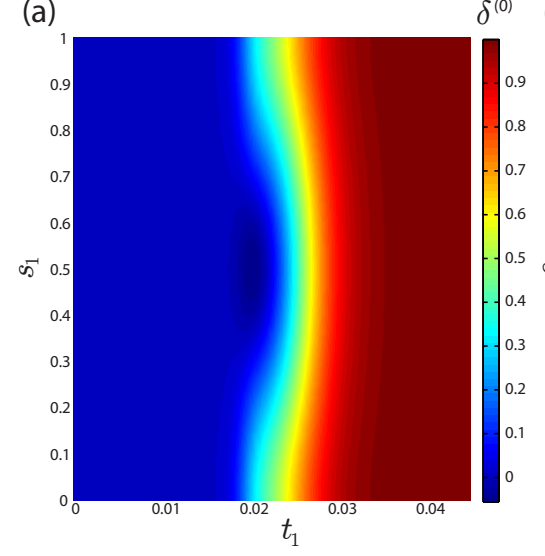

(b)

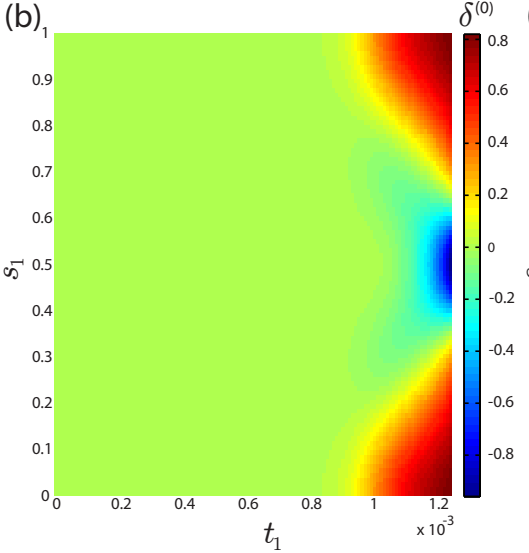

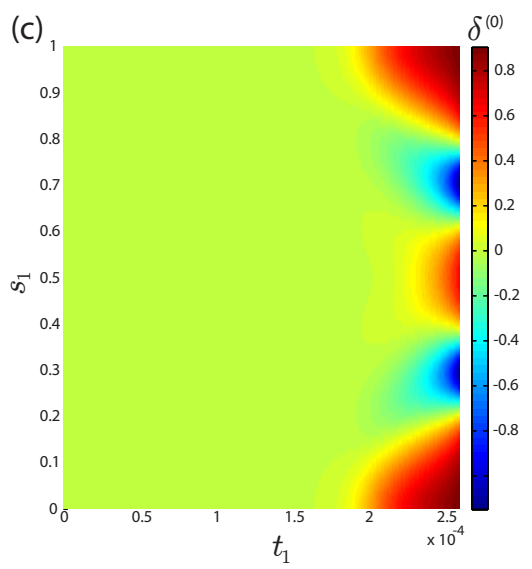

Figure 4: Numerical solutions to the reduced perturbation system $(55)-(56)$ for $\delta^{(0)}\left(0, s_{1}\right)=10^{-9}$ using a second order timeaveraging method and second order spatial finite differences with a Newton-Raphson iterative scheme. In particular: (a) $L_{1}=2 \pi$ for final time $t_{f}=4.5 \times 10^{-2}$ and time step $\Delta t_{1}=10^{-4}$; (b) $L_{1}=4 \pi$ for $t_{f}=1.25 \times 10^{-3}$ and $\Delta t_{1}=10^{-5} ;(\mathrm{c})$ $L_{1}=6 \pi$ for $t_{f}=2.6 \times 10^{-4}$ and $\Delta t_{1}=10^{-6}$. The discretized spatial grid has spacing given by $\Delta s_{1}=10^{-2}$. The final times have been chosen to show the initial pattern formation, given that $\delta^{(0)}$ is unbounded from below and will approach $-\infty$. (Color online)

(ii) We determine the protein adhesion in the new time-step by solving (32) for $c$, enforcing $\partial c / \partial s=0$ at the ends of the domain. This step does not require a Newton-Raphson iteration method because (32) is linear in $c$.

(iii) Using the updated value for $c$, we determine the new shape of the filament by solving (26)-(31) with Newton-Raphson iterations, enforcing the boundary conditions $n_{1}=n_{3}=0$ and $\kappa=c$. Steps (ii) and (iii) are repeated until the desired time is reached.

We recreate the pattern formation previously seen in Fig. 4 for the full-system by imposing a constant offset to the linearly unstable solution and solving (26)-(32) with the method outlined above for $\alpha=10^{-12}$, $\beta=10^{-12}, K_{r 0}=10^{3}, \xi=1, \xi_{\mathrm{ts}}=1, \kappa_{t}=5.5, P=10$ and $\chi=10^{10}$ which are the same parameters that generated Fig. 2. Note that $\alpha$ and $\beta$ were chosen as such to circumvent issues regarding numerical stiffness; namely, allowing for practical spacings in the discretized spatio-temporal grid. Reducing $\alpha$ and $\beta$ further does not change the plots so we conclude that that the numerical solutions have converged and that $\alpha$ and $\beta$ are still sufficiently small such that the additional filamentary mechanical contributions in the form of dampened oscillations around the bistable solutions, arising from weaker fluid drag, are not included in the computations.

For the listed parameters, $\kappa^{\star} \approx\left\{2.6996 \times 10^{-6}, 4.1790,6.4384\right\}$ by $(33)$, so we set the initial condition $\kappa(0, s)=c(0, s)=4.1791$, perturbing the unstable steady state by $10^{-4}$, and produce numerical solutions for $\kappa(t, s)$ with $D-\chi=\{50,5,0.5\}$, shown in Fig. 5(a), (b), and (c) respectively. There are a number of important aspects from Fig. 5 to comment on. First, $\kappa$ locally converges to the linearly stable states $\kappa^{\star}=\left\{2.6696 \times 10^{-6}, 6.4384\right\}$, as is evident in Fig. 5(b) and 5(c), meaning that $\kappa$ is bounded from below and remains finite for all time. Second, for $D-\chi=50, \kappa$ resolves to the spatially uniform stable solution, but as $D-\chi$ decreases to 5 and 0.5 , the shape of the filament and the protein concentration is dominated by pattern formation that resolves on longer time scales. Third, decreasing $D-\chi$ results in increasingly oscillatory patterns in $\kappa$ that occur in shorter times from the initial perturbation; patterns which share similarities with those from Fig. 4 . We noted previously that this result was due to boundary driven effects given by the condition $\mu=0$, which derives from the enforcement of zero moments acting at the ends of the filament $\kappa=c$. Indeed, from (28), this condition implies that all derivatives of $n_{1}$ vanish which suggests that all derivatives of $v_{1}$ vanish too from (26), given that $n_{3}=0$ is another boundary condition. From a combination of (29)-(31), this consequence implies that $w_{2}=0$ so that $\partial \kappa / \partial t=0$. Given that $\kappa=c$, the left hand side of (32) is zero so that, in a similar manner as (55), the second derivative of $c$ is non-zero, for small $D-\chi$, in order for this boundary condition to be satisfied. All of these aspects were predicted in the weakly nonlinear analysis of Section 3.3.

As a final illustrative example showing the full resolution of the bistable system, from initial pattern 

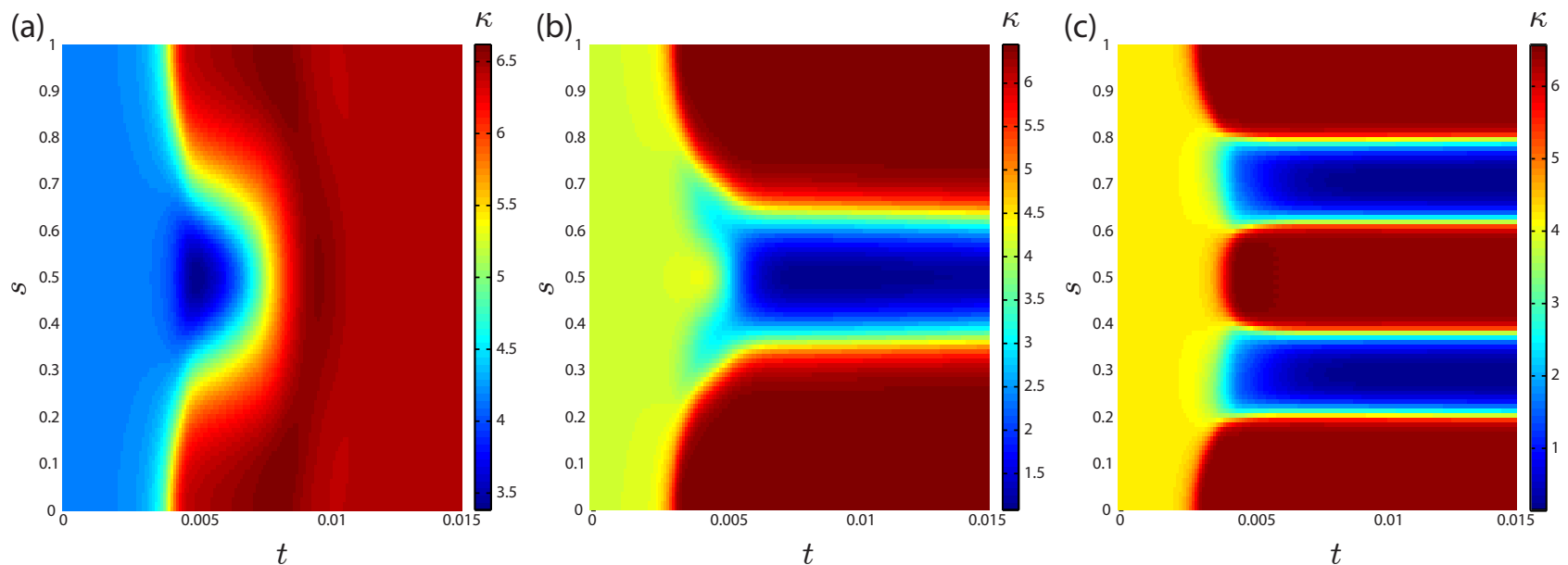

Figure 5: Numerical solutions of (26)-(32) showing $\kappa(t, s)$. An implicit time-differencing method with time-step $\Delta t=10^{-4}$ to final time $t_{f}=0.015$ and second order spatial differencing with grid spacing $\Delta s=10^{-2}$ was used for $\alpha=10^{-12}, \beta=10^{-12}$, $K_{r 0}=10^{3}, \xi=1, \xi_{\text {ts }}=1, \kappa_{t}=5.5, P=10, \chi=10^{10}$ and (a) $D-\chi=50$, (b) $D-\chi=5$, and (c) $D-\chi=0.5$. The initial conditions are $\kappa(0, s)=c(0, s)=4.1791$ and $n_{1}(0, s)=n_{3}(0, s)=v_{1}(0, s)=v_{3}(0, s)=w_{2}(0, s)=0$. (Color online)

formation to phase-coarsening, we solve (26)-(32) for the same parameters as per Fig. 5 with $D-\chi=1$ and impose random initial data with amplitude $10^{-4}$ around the unstable curvature $\kappa^{\star}=4.1790$; that is, $\kappa(0, s)=c(0, s) \in[4.1789,4.1791]$. A realization of the resultant $\kappa$ and the corresponding shape of the filament in 2D space, obtained by solving the Frenet-Serret equations, are shown in Fig. 6. The results illustrate the pattern formation, resulting from local convergence of $\kappa$ and $c$ to the stable steady states $\left\{2.6696 \times 10^{-6}, 6.4384\right\}$, which physically corresponds to proteins aggregating to form regions of high and low curvature, and slow spatially uniform resolution to the highly curved steady state due to a combination of protein recruitment and protein movement along the filament.

\section{Competing Protein Processes}

As mentioned previously, there is an in-built asymmetry in the 1D self-assembly system due to the protein recruitment term that was derived; namely, that $c \in[0, P]$ so that negative filament curvatures could never be possible. It was also implied that proteins only attach onto one side of the filament and cause its intrinsic curvature to change due to the presence of $c$ in (28). There is no reason to suggest that proteins cannot also attach onto the opposite side of the filament. We extend the formalism presented in Section 2.1 and 2.2 to include the effects of multiple BAR protein types attaching and inducing curvature on both sides of the filament and study the constant curvature solutions that arise from this system.

\subsection{Inclusion of Multiple Proteins}

We suppose the filament now divides the cellular fluid into two sections and label a positive $(+)$ and negative $(-)$ side of the filament where BAR proteins of a particular type only occupy. It is assumed that no protein mixing occurs at the boundary between the positive and negative regions of the fluid. We only consider proteins which induce positive curvature upon attaching to the positive side of the filament and negative curvature attaching to the negative side, and not the presence of inverse proteins, such as the IBAR, which induce curvature in an opposite manner [14], though such proteins can be included in the model. Proteins attaching onto the positive side of the filament see the curvature as being $\kappa$ whilst those attaching onto the negative side see the curvature $-\kappa$. Lastly, we assume that the movement of a particular species along the filament occurs independently of all other attached BAR protein types; any interaction between proteins is mediated through $\kappa$.

Suppose that we have $N_{+}$different types of proteins attaching to the positive side and $N_{-}$types attaching to the opposing negative side. The number of proteins per unit length of the $i^{\text {th }}$ type on the positive side is $c_{i}^{+}$ while the associated characteristic protein parameters (i.e. its target curvature, persistence length, etc.) are 

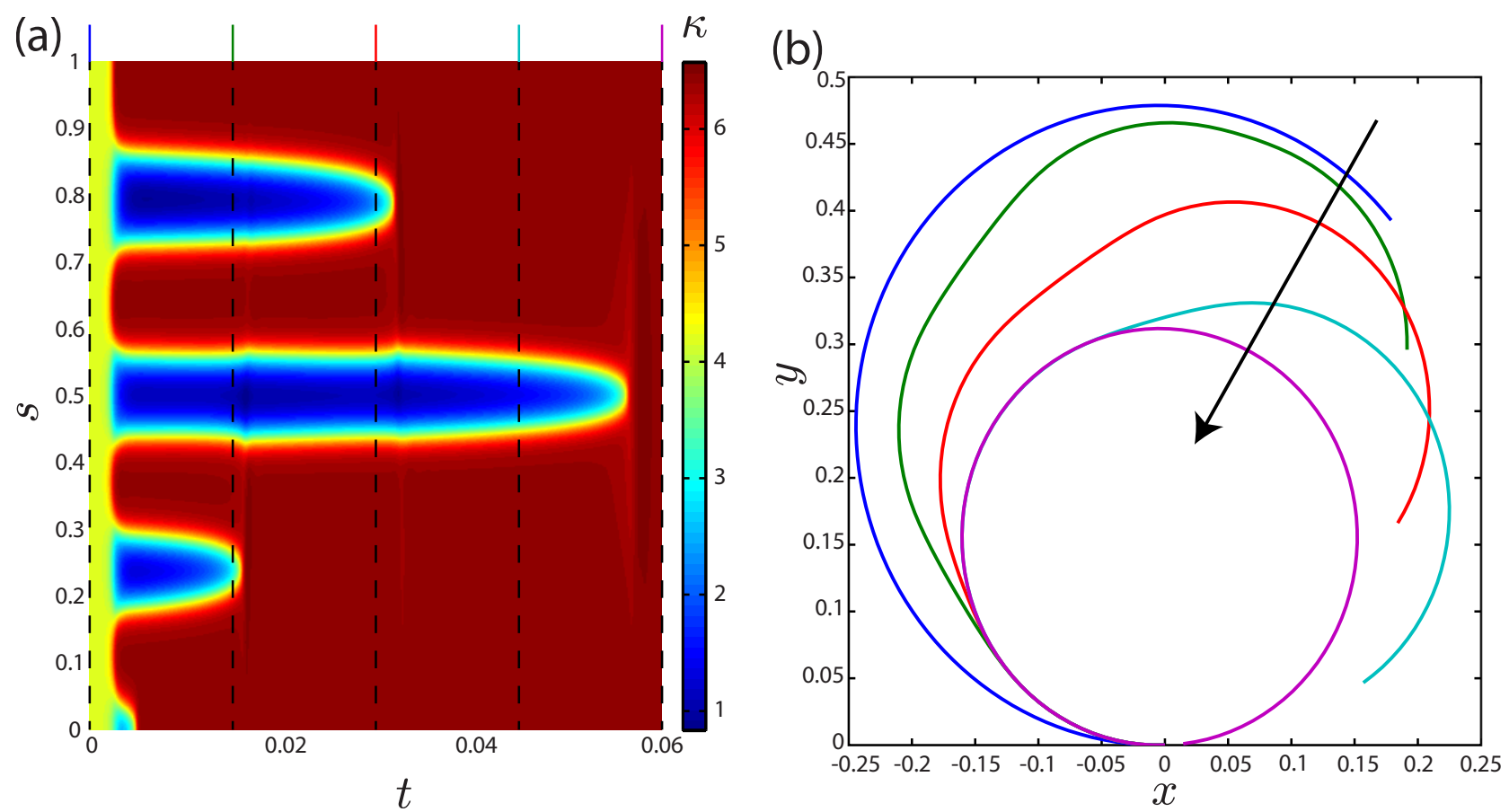

Figure 6: Time-evolution of the curvature profile $\kappa(t, s)$ (shown in (a)) and the corresponding shape of the filament in 2D space at $t=\{0,0.015,0.03,0.045,0.06\}$ (shown in (b)), resulting from the numerical solutions of (26)-(32) with time-step $\Delta t=5 \times 10^{-5}$ to final time $t_{f}=0.06$ and grid spacing $\Delta s=10^{-2}$. In particular, the parameters $\alpha=10^{-12}, \beta=10^{-12}$, $K_{r 0}=10^{3}, \xi=1, \xi_{\mathrm{ts}}=1, \kappa_{t}=5.5, P=10, \chi=10^{10}$, and $D-\chi=1$ were used, together with random initial data $\kappa(0, s)=c(0, s) \in[4.1789,4.1791]$ and $n_{1}(0, s)=n_{3}(0, s)=v_{1}(0, s)=v_{3}(0, s)=w_{2}(0, s)=0$. Arrow corresponds to increasing time. (Color online)

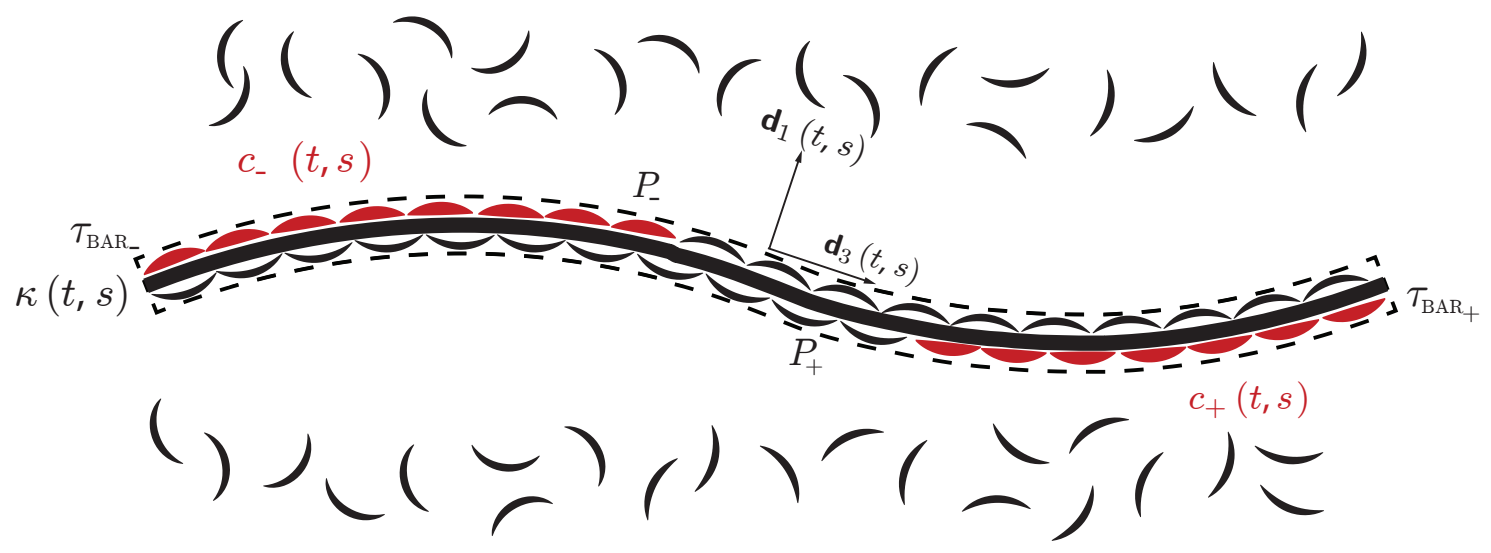

Figure 7: The extended 1D self-assembly model. Proteins are recruited to the positive side, as with the single protein case, but also on the opposite side, which is denoted as being negative. The associated characteristic protein parameters for a particular species have $(+)$ or $(-)$ subscripts depending on the side to which they attach. (Color online) 
generally given with the superscript and subscript $\Omega_{i}^{+}$for $\Omega=\left\{\gamma, \tau_{\mathrm{BAR}}, K_{r 0}, K_{\text {eqm }}, K_{\text {int }}, \xi, \xi_{\text {ts }}, \kappa_{t}, P, D, \chi\right\}$. Similarly, the concentration of the $j^{t h}$ type of proteins on the negative side is $c_{j}^{-}$with characteristic protein parameters $\Omega_{j}^{-}$(see Fig. 7). For the forthcoming analysis, all variables will be taken to be nondimensionalized in the same manner as in Section 2.3.

Since the sign of the resulting curvature the proteins induce on the filament depends on the side to which they have attached, the equations describing the mechanics of the filament in (26)-(27) and (29)-(31) remain the same except for (28), which becomes:

$$
\beta \frac{\partial w_{2}}{\partial t}=\frac{\partial}{\partial s}\left(\kappa-\sum_{i=1}^{N_{+}} c_{i}^{+}+\sum_{j=1}^{N_{-}} c_{j}^{-}\right)+n_{1},
$$

with free-ends boundary conditions given by $n_{1}=n_{3}=0$ and:

$$
\kappa=\sum_{i=1}^{N_{+}} c_{i}^{+}-\sum_{j=1}^{N_{-}} c_{j}^{-},
$$

applicable at $s=\{0,1\}$.

The effect of an inverse protein, which induces negative curvature upon attaching onto the positive side of the filament, is equivalent to a non-inverse protein that induces negative curvature by attaching to the negative side of the filament. As such, including the effects of proteins like the I-BAR is a matter of changing the sign of its induced curvature and attaching it to the opposite side of the filament, which can be encoded in (60).

The equation describing the attachment and movement of a particular protein species on the positive side of the filament is the same as for the single protein type:

$$
\begin{aligned}
\frac{\partial c_{i}^{+}}{\partial t}=K_{r 0_{i}}^{+} \exp \left(\frac{1}{2}\left(\xi_{i}^{+}-\xi_{\mathrm{ts}_{i}}^{+}\right)\left(\kappa-\kappa_{t_{i}}^{+}\right)^{2}\right)\left\{P_{i}^{+} \exp \left(-\frac{\xi_{i}^{+}}{2}\left(\kappa-\kappa_{t_{i}}^{+}\right)^{2}\right)-c_{i}^{+}\right\} & \\
& +D_{i}^{+} \frac{\partial^{2} c_{i}^{+}}{\partial s^{2}}+\chi_{i}^{+} \frac{\partial}{\partial s}\left[c_{i}^{+} \frac{\partial}{\partial s}\left(\kappa-\kappa_{t_{i}}^{+}\right)^{2}\right],
\end{aligned}
$$

with no flux boundary conditions $\partial c_{i}^{+} / \partial s=0$ at $s=\{0,1\}$. However, for the negative side of the filament, attaching proteins would see the opposite sign of filament curvature $-\kappa$, so that:

$$
\begin{aligned}
\frac{\partial c_{j}^{-}}{\partial t}=K_{r 0_{j}}^{-} \exp \left(\frac{1}{2}\left(\xi_{j}^{-}-\xi_{\mathrm{ts}_{j}}^{-}\right)\left(-\kappa-\kappa_{t_{j}}^{-}\right)^{2}\right)\left\{P_{j}^{-} \exp \left(-\frac{\xi_{j}^{-}}{2}\left(-\kappa-\kappa_{t_{j}}^{-}\right)^{2}\right)-c_{j}^{-}\right\} & \\
& +D_{j}^{-} \frac{\partial^{2} c_{j}^{-}}{\partial s^{2}}+\chi_{j}^{-} \frac{\partial}{\partial s}\left[c_{j}^{-} \frac{\partial}{\partial s}\left(-\kappa-\kappa_{t_{j}}^{-}\right)^{2}\right],
\end{aligned}
$$

with $\partial c_{j}^{-} / \partial s=0$ at $s=\{0,1\}$.

With the addition of multiple protein processes, the self-assembly model now becomes a system of $N_{+}+$ $N_{-}+6$ coupled partial differential equations with equations for added proteins being derived by using either (62) or (63) with the characteristic protein parameters $\left\{\gamma, \tau_{\mathrm{BAR}}, K_{r 0}, K_{\mathrm{int}}, K_{\mathrm{eqm}}, \xi, \xi_{\mathrm{ts}}, \kappa_{t}, P, D, \chi\right\}$.

The criterion for time-independent solutions now becomes more complex. As before, the steady state of the filament mechanics occurs for $v_{1}^{\star}=v_{3}^{\star}=w_{2}^{\star}=n_{1}^{\star}=n_{3}^{\star}=0$ and:

$$
\kappa^{\star}=\sum_{i=1}^{N_{+}}\left(c_{i}^{+}\right)^{\star}-\sum_{j=1}^{N_{-}}\left(c_{j}^{-}\right)^{\star},
$$

for all $s$. However, from the protein adhesion kinetics, the steady state is given by the solution of a coupled set of ordinary differential equations. In the spatially uniform case, the time-independent concentration of proteins on the positive side is:

$$
\left(c_{i}^{+}\right)^{\star}=P_{i}^{+} \exp \left(-\frac{\xi_{i}^{+}}{2}\left(\kappa^{\star}-\kappa_{t_{i}}^{+}\right)^{2}\right)
$$


and for the negative side:

$$
\left(c_{j}^{-}\right)^{\star}=P_{j}^{-} \exp \left(-\frac{\xi_{j}^{-}}{2}\left(-\kappa^{\star}-\kappa_{t_{j}}^{-}\right)^{2}\right),
$$

which means that finding the thermodynamically consistent steady states becomes a matter of solving the algebraic system of (64)-(66) for $\kappa^{\star},\left(c_{i}^{+}\right)^{\star}$, and $\left(c_{j}^{-}\right)^{\star}$.

\subsection{Linear Stability Analysis}

As per Section 3.2, we linearize around the spatially uniform steady states by using the ansatz $h=$ $h^{\star}+\delta_{h} \exp (i k s+\sigma t)$ for $h=\left\{v_{1}, v_{3}, w_{2}, \kappa, n_{1}, n_{3}, c_{i}^{+}, c_{j}^{-}\right\}$where the terms $\kappa^{\star},\left(c_{i}^{+}\right)^{\star}$, and $\left(c_{j}^{-}\right)^{\star}$ satisfy (64)(66). Upon substitution into (26)-(27), (60), and (29)-(31) in the limit $\alpha, \beta \rightarrow 0^{+}$, we again find that all $\delta_{j}$ for $j=\left\{v_{1}, v_{3}, w_{2}, n_{1}, n_{3}\right\}$ can be expressed in terms of $\delta_{\kappa}, \delta_{c_{i}^{+}}$, and $\delta_{c_{j}^{-}}$to linear order, so that:

$$
\sigma \delta_{\kappa}=-H \delta_{\kappa}+H\left(\sum_{i=1}^{N_{+}} \delta_{c_{i}^{+}}-\sum_{j=1}^{N_{-}} \delta_{c_{j}^{-}}\right),
$$

with $H=2 k^{2}\left(k^{2}-\kappa^{\star 2}\right)^{2} /\left(2 k^{2}+\kappa^{\star 2}\right)$ as before.

Applying a similar treatment to proteins attaching on the positive and negative side of the filament:

$$
\begin{aligned}
& \sigma \delta_{c_{i}^{+}}=F_{i}^{+} \delta_{\kappa}-G_{i}^{+} \delta_{c_{i}^{+}}, \\
& \sigma \delta_{c_{j}^{-}}=F_{j}^{-} \delta_{\kappa}-G_{j}^{-} \delta_{c_{j}^{-}},
\end{aligned}
$$

where:

$$
\begin{aligned}
F_{i}^{+} & =A_{i}^{+} B_{i}^{+} \xi_{i}^{+} P_{i}^{+}\left(\kappa_{t_{i}}^{+}-\kappa^{\star}\right)-2 \chi_{i}^{+}\left(c_{i}^{+}\right)^{\star}\left(\kappa^{\star}-\kappa_{t_{i}}^{+}\right) k^{2}, \\
G_{i}^{+} & =A_{i}^{+}+D_{i}^{+} k^{2}, \\
F_{j}^{-} & =A_{j}^{-} B_{j}^{-} \xi_{j}^{-} P_{j}^{-}\left(\kappa^{\star}+\kappa_{t_{j}}^{-}\right)+2 \chi_{j}^{-}\left(c_{j}^{-}\right)^{\star}\left(\kappa^{\star}+\kappa_{t_{j}}^{-}\right) k^{2}, \\
G_{j}^{-} & =A_{j}^{-}+D_{j}^{-} k^{2},
\end{aligned}
$$

with:

$$
\begin{aligned}
& A_{i}^{+}=K_{r 0_{i}}^{+} \exp \left(\frac{1}{2}\left(\xi_{i}^{+}-\xi_{\mathrm{ts}_{i}}^{+}\right)\left(\kappa^{\star}-\kappa_{t_{i}}^{+}\right)^{2}\right)>0, \\
& B_{i}^{+}=\exp \left(-\frac{\xi_{i}^{+}}{2}\left(\kappa^{\star}-\kappa_{t_{i}}^{+}\right)^{2}\right)>0, \\
& A_{j}^{-}=K_{r 0_{j}}^{-} \exp \left(\frac{1}{2}\left(\xi_{j}^{-}-\xi_{\mathrm{ts}_{j}}^{-}\right)\left(-\kappa^{\star}-\kappa_{t_{j}}^{-}\right)^{2}\right)>0, \\
& B_{j}^{-}=\exp \left(-\frac{\xi_{j}^{-}}{2}\left(-\kappa^{\star}-\kappa_{t_{j}}^{-}\right)^{2}\right)>0 .
\end{aligned}
$$

An equation incorporating (67)-(69) can be written so that the linear stability of the spatially homogeneous solutions is found by determining the eigenvalues of a $\left(N_{+}+N_{-}+1\right) \times\left(N_{+}+N_{-}+1\right)$ matrix:

$$
\sigma\left[\begin{array}{c}
\delta_{\kappa} \\
\delta_{c_{1}^{+}} \\
\vdots \\
\delta_{c_{N_{+}}^{+}} \\
\delta_{c_{1}^{-}}^{-} \\
\vdots \\
\delta_{c_{N_{-}}^{-}}^{-}
\end{array}\right]=\left[\begin{array}{ccccccc}
-H & H & \cdots & H & -H & \cdots & -H \\
F_{1}^{+} & -G_{1}^{+} & 0 & \cdots & \cdots & \cdots & 0 \\
\vdots & 0 & \ddots & \ddots & \ddots & \ddots & \vdots \\
F_{N_{+}}^{+} & \vdots & \ddots & -G_{N_{+}}^{+} & \ddots & \ddots & \vdots \\
F_{1}^{-} & \vdots & \ddots & \ddots & -G_{1}^{-} & \ddots & 0 \\
\vdots & \vdots & \ddots & \ddots & \ddots & \ddots & 0 \\
F_{N_{-}}^{-} & 0 & \cdots & \cdots & 0 & 0 & -G_{N_{-}}^{-}
\end{array}\right]\left[\begin{array}{c}
\delta_{\kappa} \\
\delta_{c_{1}^{+}} \\
\vdots \\
\delta_{c_{N_{+}}^{+}} \\
\delta_{c_{1}^{-}} \\
\vdots \\
\delta_{c_{N_{-}}^{-}}^{-}
\end{array}\right] .
$$


We can solve (78) analytically for the case $G_{1}^{+}=\cdots=G_{N_{+}}^{+}=G_{1}^{-}=\cdots=G_{N_{-}}^{-}=G$, which corresponds to the species of proteins having the same chemical and diffusion characteristics but being introduced around the filament with different concentrations $P$. We define the difference of protein adhesion contributions on the positive and negative side of the filament:

$$
F_{\text {diff }}=\sum_{i=1}^{N_{+}} F_{i}^{+}-\sum_{j=1}^{N_{-}} F_{j}^{-} .
$$

In this instance, the characteristic polynomial for $\sigma$ becomes:

$$
0=(\sigma+G)^{\left(N_{+}+N_{-}-1\right)}\left[\sigma^{2}+(G+H) \sigma+H\left(G-F_{\text {diff }}\right)\right] .
$$

We note that (80) has the same form as (41) such that, in the limit of $k \rightarrow 0^{+}$, instabilities would also arise. Indeed, the instability criterion shares a similar form as for single protein species driven assembly:

$$
F_{\text {diff }}^{0}-A>0,
$$

where $F_{\mathrm{diff}}^{0}=\lim _{\chi_{i}^{+}, \chi_{j}^{-} \rightarrow 0} F_{\mathrm{diff}}$ and $A$ is defined in (38) and is the same for the case of chemically similar proteins.

Equation (81) can be interpreted physically. In Section 3.2, we found that the adhesion kinetics of one protein species was the driving process of instabilities for filament curvatures less than the protein target curvature. Criterion (81) suggests that when multiple proteins are introduced, it is the combined contributions of the adhesion kinetics on both sides of the filament that determine whether a particular solution is unstable. For positive $\kappa^{\star}$ that is unstable, proteins recruited on the positive side of the filament act as a means of destabilization, whilst those attaching to the negative side stabilize. The opposite applies for negative $\kappa^{\star}$, given the $-\kappa^{\star}$ factor in $F_{j}^{-}$. In other words, the competition of stabilizing and destabilizing proteins on opposing sides of the filament determines the overall stability of the filament's shape.

For protein species with different values of $G$, the eigenvalues of the matrix in (78) do not allow for a simple form of solution due to coupling between $G_{i}^{+}$and $G_{j}^{-}$terms; the adhesion interactions mediated by the curvature of the filament play a higher role in determining the stability of the final self-assembled structure and can therefore only be solved under limiting cases or numerically.

\section{Conclusion}

In the present work, we considered a simplified model for the self-assembly of a 1D continuum by means of curvature inducing regions modeling the effect of BAR proteins in 1D. The main motivation of this model is to determine the role that interactions between the filamentary mechanics and the protein adhesion kinetics play in the resultant shapes. In particular, we considered an inextensible, unshearable planar Kirchhoff rod that was freely floating in a fluid and coupled its intrinsic curvature to the number of proteins attached to it. We then derived a time-evolution equation for protein recruitment, that was consistent with the microscopic bending energy of an individual protein, and considered the effects of thermal diffusion and drift to minimize the protein bending energy.

We studied the conditions under which time-independent solutions arose, focusing primarily on those which were spatially uniform. In this instance, the filament mechanics was not sufficient to determine the steady state of the self-assembled system; the thermodynamics of protein adhesion constrained the solutions according to the characteristic parameters of the protein. Furthermore, we established that these same parameters completely defined the corresponding linear stability under long and short wave perturbations. Proteins could act as a means of destabilizing the shape of the filament. For increasing persistence lengths of the attaching proteins, which corresponds to lower temperature environments or proteins with greater mechanical stiffness, there was a single globally stable high curvature solution up until a critical persistence length, whereby a saddle-node bifurcation appeared introducing bistable high and low curvature solutions and a middle unstable solution.

Using weakly nonlinear analysis in the long wave limit, we demonstrated that perturbing the unstable curvature resembled a Cahn-Hilliard type problem; proteins would aggregate to form regions of high and low 
curvature which interacted and merged to form a stable spatially uniform filament shape. The time-scale in which this coarsening occurred was inversely dependent on the effective motion of proteins, with thermal diffusion acting as a means of smoothing the pattern and drift serving to maintain it. However, we showed that even perturbing the unstable state with a constant offset could result in pattern formation in the protein concentration due to the boundary conditions imposed on the filament; namely, that there were no applied moments at its ends.

We extended the self-assembly model to study the effects of multiple protein types attaching to both sides of the filament. The criterion of time-independent solutions in this instance was more complicated due to the additional thermodynamic inclusions from protein recruitment, all of which was mediated through the curvature of the filament. A means of determining the linear stability of spatially uniform solutions was outlined and analytically solved for the case of chemically similar protein species with the same diffusion constants being introduced around the filament with different maximum concentrations. Each individual protein type contributed to the stability of the final filament shape. Whereas in the single protein species case whereby proteins could act as a means of destabilization, we established that protein types attaching to the opposite side of the filament stabilized the shape. Proteins on opposing ends competed to determine the overall stability of the filament curvature.

It should be clear that any simplified 1D model misses some behaviors of actual lipid membranes. In particular, since the elastic rod can conform to the protein curvature, it does not induce change of curvature in the entire structure as found in actual curvature-inducing proteins. Nevertheless, these simple models are particularly useful to understand the interplay between protein kinetics, membrane curvature, and selfassembly.

\section{References}

[1] S. S. Antman. Nonlinear Problems of Elasticity. Springer, New York, 2nd edition, 2005.

[2] A. Arkhipov, Y. Yin, and K. Schulten. Four-scale description of membrane sculpting by BAR domains. Biophysical Journal, 95(6):2806-2821, September 2008.

[3] A. Arkhipov, Y. Yin, and K. Schulten. Membrane-bending mechanism of amphiphysin N-BAR domains. Biophysical Journal, 97(10):2727-2735, November 2009.

[4] G. S. Ayton, E. Lyman, and G. A. Voth. Hierarchical coarse-graining strategy for protein-membrane systems to access mesoscopic scales. Faraday discussions, 144:347-481, 2010.

[5] F. Campelo, H. T. McMahon, and M. M. Kozlov. The hydrophobic insertion mechanism of membrane curvature generation by proteins. Biophysical Journal, 95(5):2325-2339, September 2008.

[6] B. R. Capraro, Y. Yoon, W. Cho, and T. Baumgart. Curvature sensing by the Epsin N-Terminal Homology domain measured on cylindrical lipid membrane tethers. Journal of the American Chemical Society, 132(4):1200-1201, February 2010.

[7] B. D. Coleman, E. H. Dill, M. Lembo, Z. Lu, and I. Tobias. On the dynamics of rods in the theory of Kirchhoff and Clebsch. Archive for Rational Mechanics and Analysis, 121(4):339-359, 1993.

[8] M. C. Cross and P. C. Hohenberg. Pattern formation outside of equilibrium. Rev. Mod. Phys., 65:8511112, July 1993.

[9] H. Cui, E. Lyman, and G. A. Voth. Mechanism of membrane curvature sensing by amphipathic helix containing proteins. Biophysical Journal, 100(5):1271-1279, March 2011.

[10] G. de Vaucouleurs. The case for a hierarchical cosmology. Science, 167(3922):1203-1213, February 1970.

[11] M. Dembo, D. C. Torney, K. Saxman, and D. Hammer. The reaction-limited kinetics of membraneto-surface adhesion and detachment. Proceedings of the Royal Society of London. Series B, Biological Sciences, 234(1274):55-83, 1988.

[12] M. Deserno. Fluid lipid membranes - a primer. 2007. 
[13] A. Frost, R. Perera, A. Roux, K. Spasov, O. Destaing, E. H. Egelman, P. De Camilli, and V. M. Unger. Structural basis of membrane invagination by F-BAR domains. Cell, 132(5):807-817, March 2008.

[14] A. Frost, V. M. Unger, and P. D. Camilli. The BAR domain superfamily: Membrane-molding macromolecules. Cell, 137(2):191-196, April 2009.

[15] J. Gerhart and M. Kirschner. Cells, Embryos and Evolution. Wiley-Blackwell, 1st edition, 1997.

[16] N. Ghoniem and D. Walgraef. Instabilities and Self-Organization in Materials: Volume II: Applications in Materials Design and Nanotechnology. Oxford University Press, 2008.

[17] F. Gittes, B. Mickey, J. Nettleton, and J. Howard. Flexural rigidity of microtubules and actin filaments measured from thermal fluctuations in shape. The Journal of Cell Biology, 120(4):923-934, February 1993.

[18] R. E. Goldstein and S. A. Langer. Nonlinear dynamics of stiff polymers. Phys. Rev. Lett., 75(6):10941097, August 1995.

[19] A. Goriely. The Mathematics and Mechanics of Biological growth. Springer, New York, 2017.

[20] J. D. Halley and D. A. Winkler. Consistent concepts of self-organization and self-assembly. Complexity, 14(2):10-17, November 2008.

[21] M. C. Heinrich, B. R. Capraro, A. Tian, J. M. Isas, R. Langen, and T. Baumgart. Quantifying membrane curvature generation of Drosophila Amphiphysin N-BAR domains. Journal of Physical Chemistry Letters, 1:3401-3406, November 2010.

[22] W. Helfrich. Elastic properties of lipid bilayers: Theory and possible experiments. Zeitschrift für Naturforschung, C: Journal of Biosciences, 28(11-12):693-703, December 1973.

[23] U. Jelerčič and N. S. Gov. Pearling instability of membrane tubes driven by curved proteins and actin polymerization. Physical Biology, 12(6):066022-, 2015.

[24] D. Kabaso, N. Bobrovska, W. Góźdź, N. Gov, V. Kralj-Iglič, P. Veranič, and A. Iglič. On the role of membrane anisotropy and BAR proteins in the stability of tubular membrane structures. Journal of Biomechanics, 45(2):231-238, January 2012.

[25] D. Kabaso, E. Gongadze, P. Elter, U. van Rienen, J. Gimsa, V. Kralj-Igliç, and A. Igliç. Attachment of rod-like (BAR) proteins and membrane shape. Mini-Reviews in Medicinal Chemistry, 11(4):272-282, 2011.

[26] A. Y. Kasumov, D. V. Klinov, P. E. Roche, S. Guéron, and H. Bouchiat. Thickness and low-temperature conductivity of DNA molecules. Applied Physics Letters, 84(6):1007-1009, 2004.

[27] J. B. Keller and S. I. Rubinow. Slender-body theory for slow viscous flow. Journal of Fluid Mechanics, 75:705-714, June 1976.

[28] R. Lipowsky. The conformation of membranes. Nature, 349:475-481, February 1991.

[29] J. L. McWhirter, G. Ayton, and G. A. Voth. Coupling field theory with mesoscopic dynamical simulations of multicomponent lipid bilayers. Biophysical Journal, 87(5):3242-3263, November 2004.

[30] C. Mim, H. Cui, J. A. Gawronski-Salerno, A. Frost, E. Lyman, G. A. Voth, and V. M. Unger. Structural basis of membrane bending by the N-BAR protein endophilin. Cell, 149(1):137-145, March 2012.

[31] S. Morlot and A. Roux. Mechanics of dynamin-mediated membrane fission. Annu. Rev. Biophys., 42(1):629-649, May 2013.

[32] D. E. Moulton, T. Lessinnes, and A. Goriely. Morphoelastic rods. Part I: A single growing elastic rod. Journal of the Mechanics and Physics of Solids, 61(2):398-427, February 2013. 
[33] B. Peleg, A. Disanza, G. Scita, and N. Gov. Propagating cell-membrane waves driven by curved activators of actin polymerization. PLoS One, 6(4):1-11, April 2011.

[34] B. J. Peter, H. M. Kent, I. G. Mills, Y. Vallis, P. J. G. Butler, P. R. Evans, and H. T. McMahon. BAR domains as sensors of membrane curvature: The amphiphysin BAR structure. Science, 303(5657):495499, January 2004.

[35] W. A. Prinz and J. E. Hinshaw. Membrane-bending proteins. Critical reviews in biochemistry and molecular biology, 44(5):278-291, 2009.

[36] W. Rawicz, K. C. Olbrich, T. McIntosh, D. Needham, and E. Evans. Effect of chain length and unsaturation on elasticity of lipid bilayers. Biophysical Journal, 79(1):328-339, July 2000.

[37] P. W. K. Rothemund. Folding DNA to create nanoscale shapes and patterns. Nature, 440:297-302, March 2006.

[38] A. B. Sendova-Franks and N. R. Franks. Self-assembly, self-organization and division of labour. Philosophical Transactions of the Royal Society of London B, 354(1388):1395-1405, August 1999.

[39] Z. Shi and T. Baumgart. Dynamics and instabilities of lipid bilayer membrane shapes. Advances in Colloid and Interface Science, 208:76-88, June 2014.

[40] Y. Shibata, J. Hu, M. M. Kozlov, and T. A. Rapoport. Mechanisms shaping the membranes of cellular organelles. Annual Review of Cell and Developmental Biology, 25:329-354, November 2009.

[41] M. Simunovic and P. Bassereau. Reshaping biological membranes in endocytosis: crossing the configurational space of membrane-protein interactions. Biological Chemistry., 395(3):275-283, March 2014.

[42] M. Simunovic, C. Mim, T. C. Marlovits, G. Resch, V. M. Unger, and G. A. Voth. Protein-mediated transformation of lipid vesicles into tubular networks. Biophysical Journal, 105(3):711-719, August 2013.

[43] M. Simunovic, A. Srivastava, and G. A. Voth. Linear aggregation of proteins on the membrane as a prelude to membrane remodeling. Proceedings of the National Academy of Sciences, 110(51):2039620401, December 2013.

[44] B. Sorre, A. Callan-Jones, J. Manzi, B. Goud, J. Prost, P. Bassereau, and A. Roux. Nature of curvature coupling of amphiphysin with membranes depends on its bound density. Proceedings of the National academy of Sciences of the United States of America, 109(1):173-178, January 2012.

[45] A. Srivastava and G. A. Voth. Hybrid approach for highly coarse-grained lipid bilayer models. J. Chem. Theory Comput., 9(1):750-765, January 2013.

[46] S. Suetsugu, S. Kurisu, and T. Takenawa. Dynamic shaping of cellular membranes by phospholipids and membrane-deforming proteins. Physiological Reviews, 94(4):1219-1248, October 2014.

[47] M. J. Tindall, P. K. Maini, S. L. Porter, and J. P. Armitage. Overview of mathematical approaches used to model bacterial chemotaxis II: Bacterial populations. Bulletin of Mathematical Biology, 70(6):1570$1607,2008$.

[48] K. Weiß, A. Neef, Q. Van, S. Kramer, I. Gregor, and J. Enderlein. Quantifying the diffusion of membrane proteins and peptides in black lipid membranes with 2-focus fluorescence correlation spectroscopy. Biophysical Journal, 105(2):455-462, July 2013.

[49] Y. Yin, A. Arkhipov, and K. Schulten. Simulations of membrane tubulation by lattices of amphiphysin N-BAR domains. Structure, 17(6):882-892, June 2009.

[50] Q. Zheng and G. W. Wei. Poisson-Boltzmann-Nernst-Planck model. The Journal of Chemical Physics, 134(19):194101-, March 2011.

[51] J. Zimmerberg and M. M. Kozlov. How proteins produce cellular membrane curvature. Nature Reviews. Molecular Cell Biology, 7(1):9-19, January 2006. 